SFRVIÇO DE POS-GRAIULAÇÃO DO ICMC-USP

Data de Deposito: $\quad 24.06 .2002$.

Assinatura:

\title{
Resultados de existência de soluções para uma equação diferencial funcional com impulsos
}

\author{
Sérgio Henrique Nogueira \\ Orientador: Prof. Dr. Eduardo Alex Hernandez Morales
}

Disscrtação apresentada ao Instituto de Ciências Matemáticas c de Computação - ICMC-USP. como parte dos requisitos para obtenção do título de Mestre cm Matemática.

USP - São Carlos

Junho/2002 


\section{A Comissão Julgadora:}

Prof. Dr. Lduardo Alex Hernandez Morales

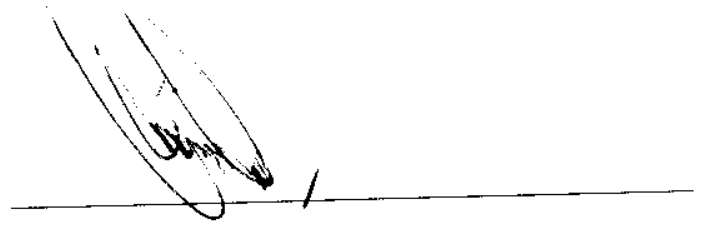

Prof. Dr. Aloisio José Freiria Neves

Profa. Dra. Sueli Mieko Tanaka Aki

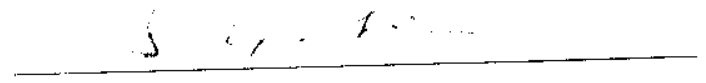


Ao pai e à măe,

Jack, Renato

e Nathália. 


\section{Agradecimentos}

A Universidade de São Paulo, em especial aos professores do ICMC-USP: Mírian P. Mendes, Roberta G. W. Atique e Valdir A. Menegatto, pela oportunidade.

A CAPES, pelo apoio financeiro.

Ao meu orientador Eduardo A. Hernández Morales, que paciontemente me ajudou a vencer as dificuldaes e me orienton com exomplar competência e dedicação.

Aos colegas do Instituto: Alexandre P. Calixto, Cláudia R. Lima, Daniel W. Mancini, Elíris C. Riziolli, Esdras T. Costa, Hildebrane A. dos Santos, Juciano N. Morcira e Karina Schiabell, que, da convivencia, se tornaram amigos e tanto ajudaram.

Aos amigos de sempre: César Augusto V. Passos, Eufrásio A. C. Júnior, Josemauro Alves, Márcio F. da Silva e Wollington Pereira, companheiros do tantas histórias.

Mais que agradecer, eu divido este trabalho con a minha familia, especialmente: meu pai, minha màe, Antonio Wilson de O. Malta, Benisa N. Rodirgues, Inês R. Nogueira, Jaqueline Nogueira, Renato Nogueira e Sandra C. Nogueira, pelo cuidado que têm comigo. E com a Nathália, que ainda me chama de pai, apesar da ausència. Valen! Esta alegria é nossa! 


\section{Abstract}

In this work, we prove the existence of mild solutions for a class of partial functional differential equations with impulses modelled in the form

$$
\begin{array}{rlrl}
\dot{u}(t) & =A u(t)+f(t, u(t), u(a(t))), & & t \in I=[0, T], \\
u(0) & =u_{0}, & \\
\Delta u\left(t_{i}\right) & =I_{i}\left(u\left(t_{i}\right)\right), \quad i \in\{1, \ldots, n\}, & 0<t_{1}<t_{2}<\ldots<t_{n}<T,
\end{array}
$$

where $\mathrm{A}$ is the infinitesimal generator of a strongly continuous semigroup of bounded lincar operators on a Banach space $X, 0<t_{1}<t_{2}<\ldots<t_{n}<T ; f(\cdot), a(\cdot), I_{i}(\cdot), i=1,2, \ldots n$, are appropriated functions and $\Delta u\left(t_{i}\right)$ denote the jump of a function $u(\cdot)$ in the point $t_{i}$, that is $\triangle u\left(t_{i}\right)=u\left(t_{i}^{\dagger}\right)-u\left(t_{i}^{-}\right)$. 


\section{Resumo}

Neste trabalho, provamos a existência de soluçōes para uma classe de equações diferenciais com impulsos modeladas na forma

$$
\begin{array}{rlrl}
\dot{u}(t) & =A u(t)+f(t, u(t), u(a(t))), & & t \in I=[0, T], \\
u(0) & =u_{0}, & \\
\Delta u\left(t_{i}\right) & =I_{i}\left(u\left(t_{i}\right)\right), \quad i \in\{\mathbf{1}, \ldots, n\}, & 0<t_{1}<t_{2}<\ldots<t_{n}<T,
\end{array}
$$

onde $A$ é o gerador infinitesimal de um semigrupo fortemente contínuo de operadores lineares limitados $\mathrm{cm}$ um espaço de Banach $X, f(\cdot), a(\cdot), I_{i}(\cdot), i=1,2, \ldots n$, são funçôes apropriadas e $\Delta u\left(t_{i}\right)$ ć o impulso de uma função $u(\cdot)$ no ponto $t_{i}$, isto é, $\Delta u\left(t_{i}\right)=u\left(t_{i}^{+}\right)-u\left(t_{i}^{-}\right)$. 


\section{Sumário}

Introdução 1

1 Semigrupos de operadores lineares e o problema de Cauchy abstrato 3

1.1 Semigrupos de operadores lineares . . . . . . . . . . . . . . . 3

1.2 O problema de valor inicial homogêneo . . . . . . . . . . . . 13

2 O problema de Cauchy abstrato não homogêneo $\quad 17$

2.10 problema de valor inicial näo homogêneo . . . . . . . . . . . . 17

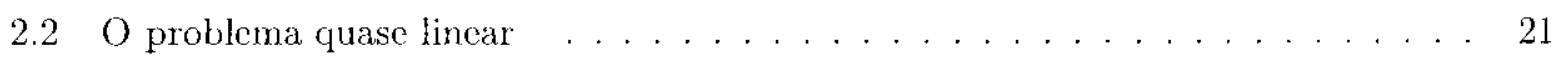

3 Um problema de evolução com impulsos $\quad 31$

3.1 Existência de soluçôes fracas . . . . . . . . . . . . . . . . 32

3.2 Existência de soluçôes globais . . . . . . . . . . . . . . . . . 40

3.3 Um exemplo: A equação do calor com impulsos . . . . . . . . . . . . . . 48 


\section{Introdução}

Neste trabalho estabclecemos alguns resultados novos de existência de soluçôes fracas para uma classe de equações diferenciais funcionais com impulsos, modeladas na forma

$$
\begin{array}{rlrl}
\dot{u}(t) & =A u(t)+f(t, u(t), u(a(t))), & & t \in I=[0, T], \\
u(0) & =u_{0}, & \\
\Delta u\left(t_{i}\right) & =I_{i}\left(u\left(t_{2}\right)\right), \quad i \in\{1, \ldots, n\}, & 0<t_{1}<t_{2}<\ldots<t_{n}<T,
\end{array}
$$

onde $A$ é o gerador infinitesimal de um semigrupo fortemente contínuo de operadores lineares limitados em um espaço de Banach $X$ e $f(\cdot), a(\cdot), I_{i}(\cdot)$ são funções apropriadas.

A teoria de equaçōes impulsivas tern recevido grande atençâo nos últimos anos devido fundamentalmente à apariçăo de várias aplicaçōos em diversas áreas, como mecânica, cugcnharia elétrica, teoria de controle, medicina e biologia. Uma referencia apropriada a respeito do tema é Rogovchenko [6], além das referências contidas nesse trabalho.

O problema (1)-(3) foi estudado recentemente, no caso em que $a(t)=t$ para $t \in I$; por Liu em [3]. Nesse artigo, Liu estabelece alguns resultados de existencia de soluçóes fracas e clássicas para o problena (1)-(3), usando o princípio de ponto fixo para contrações e as idéias do Teorema 6.1.5 em Pazy [5].

Motivados pelo artigo de Liu [3], neste trabalho cstabelecemos novos resultados de existência de soluçoes para o problema (1)-(3). Os resultados são obtidos fazendo uso da teoria de semigrupos de operadores lineares e de alguns teoremas de ponto fixo para operadores condensantes.

Este trabalho contém três capítulos. No capítulo 1, estudamos algunas propriedades básicas da teoria de semigrupos de operadores lincares limitados cm espaços de Banach; em particular provamos o clássico Teorema de Hille-Yosida para semigrupos de contraçöes. No capítulo 2 , 
estudamos a existência de soluçoes fracas, fortes e clássicas para o problema de evoluçăo

$$
\begin{aligned}
& \dot{u}(t)=A u(t)-f(t, u(t)), \quad \quad t \in I=[0, T] \\
& u(0)=u_{0} \in X .
\end{aligned}
$$

onde $A$ é o gerador infinitesimal de um $C_{0}$-semigrupo de operadores lineares limitados enı um espaço de Banach $X$. A principal referencia usada nos dois primeiros capítulos é Pazy [5]. Finalmente, no capíbulo 3 estabeleccmos novos resultados de existência de soluçóes fracas pará (1)-(3). Destacamos os Teoremas 3.5 e 3.6 a respeito de soluçôes fracas de (1))-(3) e o 'Teorema 3.10, no qual mostramos a existência de soluções globais, isto é, soluçōes definidas em $[0, \infty)$, para um problema com infinitos tempos de inpulsos. Na prova dos teormas mencionados, valemo-nos dos Teoremas 3.4 e 3.9, também inćditos. 


\section{Capítulo 1}

\section{Semigrupos de operadores lineares e o problema de Cauchy abstrato}

Em todo este trabalho adotaremos o scguinte: $X$ é um espaço de Banach real, $L(X)$ é o espaço das transformaçōes lineares contínuas definidas em $X$ e com valores em $X$ e ainda $B_{r}\left(x_{1}, Z\right)=\left\{x \in Z:\left\|x-x_{1}\right\| \leq r\right\}$, onde $B$ é espaço métrico.

Neste capítulo introduzimos alguns conceitos e propriedades básicas da teoria de semigrupos de operadores lineares em espaços de Banach, tendo como objetivo mostrar o clássico teorema de Hille-Yosida.

\subsection{Semigrupos de operadores lineares}

Definição 1.1. Uma familia de operadores lineares $(T(t))_{t \geq 0} \subset L(X)$ é um semigrupo de operadores lineares limitudos em $X$ se:

(i) $T(0)=I, \quad(I$ é o operador identidade em $X)$;

(ii) $T(t+s)=T(t) T(s), \quad$ para todo $t, s \geq 0$.

Definiçāo 1.2. Seja $(T(t))_{t \geq 0}$ um semigrupo de operadores lineares limitados em $X$. Dizemos que:

(a) $(T(t))_{t \geq 0}$ é uniformemente continuo se $\lim _{t \downarrow 0}\|T(t)-I\|_{L(X)}=0$

(b) $(T(t))_{t \geq 0}$ é fortemente contínuo, ou um $C_{0}^{\prime}$-semigrupo, se $\lim _{t \downarrow 0} T(t) x=x$, para todo $x \in X$. 
Definição 1.3. Sejam $\left(T^{\prime}(t)\right)$ @o um scmigrupo de operadores lineares limitados em $X$ e $D(A)$ o conijunto

$$
D(A)=\left\{x \in X: \lim _{t \downarrow 0} \frac{T(l) x-x}{t} \text { existe }\right\} .
$$

O operador linear $A: D(A) \subset X \rightarrow X$ definido por

$$
A x=\lim _{t, 0} \frac{T(t) x-x}{t}=\left.\frac{d^{+}}{d t} T(t) x\right|_{t-t)}, \quad x \in D(A)
$$

é o gerador infinitesimal do semigrupo $(T(t))_{1 \geq 0}$.

Todo semigrupo fortemente contínuo tem a propriedade de ser exponencialmente limitado, como mostra o próximo tcorema.

Teorema 1.4. Seja $(T(t))_{t>0}$ um semigrupo fortemente contínuo de operadores lineares em $X$. Entĩo existem ronstantes $\omega \geq 0 \circ M \geq 1$ tais que $\|T(t)\| \leq M e^{\omega \prime \prime}$, para todo $t \geq 0$.

Demonstração. Seja $\alpha>0$. Como $(T(t))_{Q_{0}}$ é fortemente contínuo, para todo $x \in X$ o conjunto $\{T(l) x: t \in[0, \alpha]\}$ é limitado em $X$, o que, pelo Teorema da limitação uniforme, implica que $\{T(t): t \in[0, \alpha]\}$ é limilado $\mathrm{cm} L(X)$. Fixemos $M \geq 1$ tal que $\|T(t)\| \leq M$ para todo $t \in[0, \alpha]$ e definamos $\omega=\alpha^{-1} \log M \geq 0$. Se $t \geq 0$ então $t=n \alpha+\beta$ para algum $0 \leq \beta<\alpha_{0}$, de onde conchúmos que $\|T(t)\|=\left\|T(\beta) T(\alpha)^{n}\right\| \leq M^{n-1} \leq M M^{\frac{t}{\alpha}}=M e^{\text {itt. }}$. Isto completa a demostração.

Definiçāo 1.5. Seja $(T(t))_{\ell \geq 0}$ um semigrupo fortemente contínuo de operudores lineares cm $X$, com $\|T(t)\| \leq M e^{\omega t}$. Dizemos que $(T(t))_{t} \geq 0$ é um semigrupo uniformemente limitado se $\omega=0$; e se, além disso $M=1$, dizemos que $(T(1))_{t>0}$ é um $C_{0}$-semigrupo de contraçoes.

Mostramos agora algumas relações básicas entre o semigrupo e o scu gerador infinitesimal.

Teorema 1.6. Sejam $(T(t))_{t \geq 0}$ um semigrupo fortemente continuo de operadores lineares limitados em $X$ e. A seu gerador infinitesimal. Entäo as seguintes propriedades são verificadas:

(a) Para todo $x \in X$ et $\geq 0$,

$$
\lim _{h \rightarrow 0} \frac{1}{h} \int_{t}^{t+h} T(s) x d s=T(t) x
$$

(b) Para todo $x \in X e t \geq 0, \int_{0}^{t} T(s) x d s \in D(A) e$

$$
A \int_{0}^{t} T(s) x d s=T(t) x-x
$$


(c) Para todo $x \in D(A), T(1) x \in D(A)$ e

$$
\frac{d}{d t} T(t) x=A T(t) x=T(t) A x
$$

(d) Para todo $x \subset D(A)$,

$$
T(1)_{x}-T(s) x=\int_{s}^{t} T(r) A x d r=\int_{s}^{t} A T(r) x d r .
$$

Demonstração. Mostramos cada propriedade de forma separada.

(a) Sejam $x \in X, t \geq 0$ e $\epsilon>0$. Como $(T(t))_{t \geq 0}$ é fortemente contínuo, podemos fixar $\delta>0$ tal que $|t(s) x-T(t) x \vdots|<\epsilon$; quando $|s-t| \leq \delta$. Consequentemente, para $|s-t| \leq \delta$ temos

$$
\begin{aligned}
\left\|\frac{1}{h} \int_{t}^{t-h} T(s) x d s-T(t) x\right\|_{i}^{i} & =\mid \frac{1}{h} \int_{t}^{t+h}(T(s) x-T(t) x) d s \| \\
& \leq \frac{1}{h} \int_{t}^{t+h}\|T(s) x \cdots T(t) x\| d s \\
& <\epsilon,
\end{aligned}
$$

o que mostra a propriedade.

(b) Para $x \in X$ e $h>0$, temos

$$
\begin{aligned}
\frac{T(h)-I}{h} \int_{0}^{t} T(s) x d s & =\frac{1}{h} \int_{0}^{l}(T(s+h) x-T(s) x) d s \\
& =\frac{1}{h} \int_{t}^{t+h} T(s) x d s-\frac{1}{h} \int_{0}^{h} T(s) x d s,
\end{aligned}
$$

o que por (a) implica que

$$
\lim _{t \downarrow 0} \frac{T(h)-I}{h} \int_{0}^{t} T(s) x=T(t) x \cdots x .
$$

Portanto $\int_{0}^{t} T(s) x d s \in D(A)$ e $A \int_{0}^{t} T(s) x d s=T(t) x-x$.

(c) Sejam $x \in D(A)$ e $t>0$. Como para $h>0$

$$
\frac{T(t+h) x-T(t) x}{h}=\frac{T(h)-I}{h} T(t) x=T(t)\left(\frac{T(h)-I}{h}\right) x,
$$

da continuidade de $T(t)$ temos

$$
\frac{d^{+}}{d t} T(t) x=A T(t) x=T(l) A x
$$

Mais ainda, se $0<h<t$

$$
\begin{aligned}
\| \frac{T(t-h) x-T(t) x}{\cdots h} & -T(t) A x \| \\
& \leq\left\|T(t-h)\left(\frac{T(h) x-x}{h}\right)-T(t) A x\right\| \\
& \leq\left\|T(t-h)\left(\frac{T(h) x-x}{h}-A x\right)\right\|+\|T(t-h) A x-T(t) A x\| \\
& \leq\|T(t-h)\|\left\|\frac{T(h) x-x}{h}-A x\right\|+\|T(t-h) A x-T(t) A x\|,
\end{aligned}
$$


o que prova que

$$
\frac{d^{-}}{d t} T(t) x=T(t) A x
$$

pois, pelo Teorema 1.4, $\|T(s)\|_{L(X)}$ é limitado em compactos de R. A propriedade é agora conseqüiencia de (1.1) e (1.2).

(d) Seja $x \in D(A)$. Pelo item (c),

$$
T(t) x-T(s) x=\int_{s}^{t} \frac{d}{d t} T(r) x d r=\int_{s}^{t} T(r) A x d r=\int_{s}^{t} A T(r) x d r
$$

Isso completa a demonstração do teorema.

Corolário 1.7. Se A é o gerador infinitesimal de um semigrupo fortemente contínuo de operadores lineares limitados $(T(t))_{l>0} \mathrm{em} X$, entäo $A$ é linear, fechado e $D(A)$ é denso em $X$.

Demonstração. Seja $x \in X$. Pelo Teorema 1.6 sabemos que, para $t>0, \frac{1}{t} \int_{0}^{t} T(s) x d s \in D(A)$ e que $\frac{1}{l} \int_{0}^{t} T(s) x d s \rightarrow x$, quando $t \downarrow 0$. Isto mostra que $\overline{D(A)}=X$. A linearidade de $A$ é evidente. Para mostrar que $A$ é fechado, suponha que $\left(x_{n}\right)_{n \in \mathbb{N}}$ é uma seqüência em $D(A)$ tal que $x_{n_{l}} \rightarrow x$ e $\Lambda x_{n} \rightarrow y$, onde $x, y \in X$. Pelo Teorema 1.6 (d), temos

$$
T(t) x_{n}-x_{n}=\int_{0}^{t} T(s) A x_{n} d s
$$

o que implica que

$$
T(t) x-x=\int_{0}^{t} T(s) y d s .
$$

Segue de (1.3) e do Teorma 1.6 (a) que $\left.\frac{d^{+}}{d t} T(t) x\right|_{t=0}-y$. Portanto $x \in D(A)$ c $A x=y$, o que mostra que $A$ é fechado. $\Lambda$ prova está completa.

A scguir estudamos quando um operador linear $A$ ć o gerador infinitesimal de um semigrupo fortemente contínuo em $X$, o que é relevante em aplicaçoes a equações diferenciais. Inicialmente estudamos o caso mais simples em que $A$ é um operador linear limitado em $X$.

Teorema 1.8. Seja $A: D(A) \subset X \rightarrow X$ um operador linear. A é o gerador infinitesimal de um semigrupo uniformemente contínuo de operadores lineares limitados $(T(t))_{t \geq 0}$ em $X$ se, e. somente se, $A$ é um operador linear limitado.

Demonstração. Suponha que $A$ é um operador limitado. Para $n \in \mathbb{N}$ delinimos o operador linear limitado $s_{n}(t)=\sum_{i=1}^{n} \frac{(t A)^{i}}{i !}$. Como $A$ é limitado, para $m, n \in \mathbb{N}, n<m$, e $t \geq 0$ temos

$$
\left\|s_{n}(t)-s_{m}(t)\right\| \leq \sum_{i, n+1}^{m} \frac{t^{i}\|A\|^{i}}{i !},
$$


o que mostra que a sequiencia $\left(s_{n}\right)_{n \in \mathbb{N}}$ ć convergente em $C([0, T]: L(X))$. Seja $e^{t, A}=\sum_{n-0}^{\infty} \frac{(L A)^{n}}{n !}$. Afirmamos que a família $(T(t))_{\geq 0}$, onde $T(t)=e^{t / A}$, é um semigrupo uniformemente contínuo de operadores lincares limitados em $X$ e que $A$ é seu gerador infinitesimal. De fato, é sabido que $T(t+s)=T(t) T(s)$ (é somente consequência de um longo e tedioso cálculo ) c claramente $T(0)=I$. Mais ainda, segue diretamente da defirição de $\epsilon^{A t}$ que

$$
\|T(t) x-x\| \leq t\|A\| e^{\ell !} \cdot A\|\| x \|,
$$

o que demonstra que o semigrupo $(T(t))_{\geq 0}$ é unifornemente contínuo em $X$. Para finalizar esta primeira parte da demonstração, vejamos que $A$ é o gerador infinitesimal de $(T(t))_{\geq 0}$. Diretamente da definição de $(T(t))_{\geq 0}$, temos

$$
T(t) x \cdots x=t A \sum_{n-0}^{\infty} \frac{(t A)^{n}}{(n+1) !}
$$

de onde obtemos que

$$
\left\|\frac{T(t) x-x}{t}-A x\right\| \leq t\|A\|^{2} e^{t: \mid A \|}\|x\|,
$$

o que mostra que $A$ é gerador de $(T(t))_{\geq 0}$.

Suponha agora que $(T(t))_{t \geq 0}$ é um semigrupo unifomemente contínuo de operadores lineares limitados em $X$. Como a função $t \rightarrow T(t)$ é contínua com valores em $L(X)$, existe $\rho_{1}>0$ tal que, para cada $\rho<\rho_{1},\left\|I-\frac{1}{\rho} \int_{0}^{\rho} T(s) d s\right\|<1$, de onde segue que $\int_{0}^{\rho} I^{\prime}(s) d s$ é invertivel para $\rho<\rho_{1}$. Nestas condiçoes. vemos que para $\rho<\rho_{1}$

$$
\begin{aligned}
\frac{1}{h}(T(h)-I) \int_{0}^{\rho} T(s) d s & =\frac{1}{h}\left(\int_{0}^{\rho} T(s+h) d s-\int_{0}^{\rho} T(s) d s\right) \\
& =\frac{1}{h}\left(\int_{\rho}^{\rho} T(s) d s-\int_{0}^{h} T(s) d s\right),
\end{aligned}
$$

o que implica que

$$
\frac{1}{h}(T(h) \cdots I)=\left(\frac{1}{h} \int_{\rho}^{\rho+h} T(s) d s-\frac{1}{h} \int_{0}^{h} T(s) d s\right)\left(\int_{0}^{\rho} T(s) d s\right)^{-1} .
$$

Usando novamente que $(T(t))_{l \geq 0}$ é um semigrupo uniformemente contínuo, concluímos de (1.4) que

$$
A x=\lim _{h \downarrow 0} \frac{1}{h}(T(h)-I) x \cdots(T(\rho)-I)\left(\int_{0}^{\rho} T(s) d s\right)^{-1} x
$$

para todo $x \in X$. Portanto $\left.A=(T(\rho)-I) \int_{0}^{\rho} T(s) d s\right)^{-1} \in L(X)$, o que completa a prova.

Definição 1.9. Seja $B: D(B) \subset X \rightarrow X$ um operador linear. Dizemos que $\lambda \in \mathbb{C}$ está no conjunto resolvente de $B$ se $(\lambda I-B)$ é invertíl e $(\lambda I-B)^{-1} \in L(X)$. O conjunto resolvente. de $B$ será denotado por $\rho(B)$. 
Antes de provar o próxino teorema, consideramos alguns lemas técnicos que seräo úteis na sua demostração. Nestes próximos lemas, sempre $A: D(A) \subset X \rightarrow X$ é urn operador linear fecluado com $\overline{D(A)}=X$.

Lema 1.10. Suponta que $\rho(A) \supset\left(0, \infty^{\dagger}\right)$ e que $\|R(\lambda: A)\|<\frac{1}{\lambda}$ para todo $\lambda>0$. Entaio as seguintes propriedades säo válidas:

(a) $\lim _{\lambda \rightarrow \infty} \lambda R(\lambda: A) x=x$, para todo $x \in X$;

(b) Se $A_{\lambda}=\lambda A R(\lambda: A)=\lambda^{2} R(\lambda: A)-\lambda I$, então $\lim _{\lambda \rightarrow \infty} A_{\lambda} x=A x$, para todo $x \in D(A)$.

Demonstração Mostramos cada propriedade separadarnente.

(a) Se $x \in D(A)$ então

$$
\begin{aligned}
\|\lambda R(\lambda: A) x \quad x\| & =\|A R(\lambda: A) x\| \\
& =\|R(\lambda: A) A x\| \\
& \leq \frac{1}{\lambda}\|A x\|:
\end{aligned}
$$

o que mostra que $\lambda R(\lambda: A) x \rightarrow x$, quando $\lambda \rightarrow \infty$. Vejamos agora o caso geral. Seja $x \in X$. Como $D(A)$ é denso em $X$ c a propriedade é válida em $D(A)$, podemos fixar $x_{r} \in D(A)$ e $\lambda_{0}>0$ tais que

$$
\begin{aligned}
\left\|x-x_{c}\right\| & <\frac{\epsilon}{3} \\
\left\|\lambda R(\lambda: A) x_{c}-x_{c}\right\| & <\frac{\epsilon}{3},
\end{aligned}
$$

quando $\lambda>\lambda_{0}$. Nestas condições, para $\lambda>\lambda_{0}$ temos

$$
\begin{aligned}
\|\lambda R(\lambda: A) x-x\| & \leq \mid \lambda i\|R(\lambda: A)\|\left\|x-x_{\epsilon}\right\|+\left\|\lambda R(\lambda: A) x_{\epsilon}-x_{\epsilon}\right\|+\left\|x_{\epsilon}-x\right\| \\
& <\lambda \frac{1}{\lambda}\left\|x-x_{\epsilon}\right\|_{1}+\frac{2 \epsilon}{3} \\
& <\epsilon,
\end{aligned}
$$

o que prova que $\lim _{\lambda \rightarrow \infty} \lambda R(\lambda: A) x=x$, para lodo $x \in X$.

( b) É consequiência direta de (a), pois para $x \in D(A)$

$$
\lim _{\lambda \rightarrow \infty} A_{\lambda} x=\lim _{\lambda \rightarrow \infty} \lambda A R(\lambda: A) x=\operatorname{limr}_{\lambda \rightarrow \infty} \lambda R(\lambda: A) A x=A x
$$

A prova do lema está agora completa.

O Lema (1.10) motiva a seguinte definição: 
Definição 1.11. Seja $A: D(A) \subset X \rightarrow X$ um operudor linear com $\rho(A) \supset\left(0, \infty^{+}\right)$. A famílin. de operadores lineares $\left(A_{\lambda}\right)_{\lambda \geq 0}$ definida por $A_{\lambda}=\lambda A R(\lambda: A)=\lambda^{2} R(\lambda: A)-\lambda I$ é chamada a. aproximaçào de Yosida de $A$.

Lema 1.12. Suponha que $\rho(A) \supset\left(0, \infty^{+}\right)$e seja $\left(A_{\lambda}\right)_{\lambda \geq 0}$ a aproximação de Yosida de A. Entáo as seguintes propriedades são vcrificadas:

(a) Para todo $\lambda>0, A_{\lambda}$ é o gerador infinitesimal de um semigrupo uniformemente contínuo de contraçoes;

(b) Se $\left(e^{t A_{\lambda}}\right)_{1 \geq 0}$ é o semigrupo gerado $p o r \cdot A_{\lambda}$ então

$$
{ }_{1} e^{t A_{\lambda}} x-e^{t \lambda_{\mu \prime}} x\|\leq t\| A_{\lambda} x-A_{\mu} x \|_{\text {, }}
$$

para todo $x \in X$ c todo $\lambda, \mu>0$.

Demonstraçāo. Como $A_{\lambda}$ é um operador linear contínuo em $X$, segue do Teorema 1.8 que $A_{\lambda}$ é o gerador infinitesimal de um semigrupo uniformemente contínuo de operadores lineares limitados $\left(e^{t A_{\lambda}}\right)_{t \geq 0} \mathrm{em} X$. Mais ainda, como

$$
\begin{aligned}
\left\|e^{t \lambda_{\lambda}}\right\| & =\left\|e^{l\left(\lambda^{2} R(\lambda: \lambda)-\cdots I\right)}\right\| \\
& =\left\|e^{t \lambda^{2} R(\lambda: A)} e^{-t \lambda I}\right\| \\
& =e^{-t \lambda}\left\|e^{t \lambda^{2} R(\lambda: A)}\right\| \\
& \leq e^{-t \lambda} e^{t \lambda^{2} \frac{1}{\lambda}}-1
\end{aligned}
$$

conclúmos que, para cada $\lambda>0,\left(e^{l A_{\lambda}}\right)_{l>0}$ é um semigrupo de contrações ; o que prova (a).

Por outro lado, das definiçōes de $e^{t A_{\theta}}$ e $A_{0}$ temros

$$
\begin{aligned}
\| e^{l A_{\lambda} x-e^{t A_{\mu}} x \|} & =\| \int_{0}^{1} \frac{d}{d s}\left(e^{t s A_{\lambda}} e^{\left.t(1 \cdot s) A_{\mu^{\prime}} x\right) d s \|}\right. \\
& \leq \int_{0}^{1}\left\|e^{t s A_{\lambda}}\left(t A_{\lambda}\right) e^{t(1-s) A_{\mu^{\prime}}} x+e^{t s A_{\lambda}} e^{t(1 \quad s) A_{\mu}}\left(-t A_{\mu}\right) x\right\| d s \\
& \leq \int_{0}^{1} t\left\|e^{l s A_{\lambda}} e^{t(1-s) A_{\mu}}\left(A_{\lambda} x-A_{\mu} x\right)\right\| d s \\
& \leq t\left\|A_{\lambda} x-A_{\mu} x\right\| \int_{0}^{1}\left\|e^{t s \lambda_{\lambda}}\right\|\left\|e^{t(1 \quad s) A_{\mu}}\right\| d s
\end{aligned}
$$

e portanto

$$
\| e^{l A_{\lambda} x-e^{t A_{\mu}} x\|\leq t\| A_{\lambda}: t-A_{\mu} x \|}
$$

o que prova (b) e completa a demonstraçào do lema. 
Observaçāo 1.13. Daqui por diante, para um operador fechado $B: D(B) \subset X \rightarrow X$, usaremos a notação $\left[D(B)\right.$ ] para o o espaço $D(B)$ munido da norma do gráfico $|x|_{B}=\|x \mid+\| B(x) \|$.

Lema 1.14. Sejam $A: D(A) \rightarrow X$ um operador linear fechado, a $\geq 0$ e $f:[0, a] \rightarrow[D(A)]$ uma funçäo contínua. Entäo

$$
A \int_{0}^{a} f(s) d s=\int_{0}^{a} A f(s) d s
$$

Demonstração. Seja $P=\left\{s_{i}: i=1 \ldots n\right\}$ uma partição de $[0, a]$ e seja também $S\left(f, I^{\prime}\right)=$ $\sum_{s_{i} \in P} f\left(s_{i}\right)\left(s_{i+1}-s_{i}\right)$ uma clásica soma de Ricrmman de $f$. Como $f \in C([0, a]: D(A))$, temos que $A S(f, P)=S(A f, P) \rightarrow \int_{0}^{b} A f(s) d s$ se $\Delta P \rightarrow 0$. Usando agora que $A$ é fechado e que $S(f, P) \rightarrow \int_{0}^{b} f(s) d s$ se $\Delta P \rightarrow 0$, concluninos que $\int_{0}^{b} f(s) d s \in D(A)$ e que

$$
A \int_{0}^{a} f(s) d s=\int_{0}^{a} A f(s) d s
$$

o que completa a prova.

Lema 1.15. Se $A$ é o gerudor infinitesimal de um $C_{0}$-semigrupo de contraçoes $(T(t))_{t \geq 0}$ em $X$, então as seguinles propriedades são válidas:

(a) Para cada $\lambda, R(\lambda)=\int_{0}^{\infty} e^{-\lambda s} T(s) d s \in L(X)$;

(b) Se $x \in D(A)$ entĩo $A R(\lambda) x-\int_{0}^{\infty} e^{-\lambda s} T(s) A x d s$.

Demonstraçāo. Como $(T(t))_{t \geq 0}$ é um senigrupo de contraçoes, $\left\|e^{\lambda s} T(s)\right\| \leq e^{-\lambda s}$. Logo, pelo Teorema da convergência dominada de Lebesgne, $R(\lambda) \in L(X)$ para $\lambda>0$.

Por outro lado, se $x \in D(A)$, do Lema 1.14 vemos que

$$
\int_{0}^{\infty} e^{-\lambda s} T(s) A x d s=\lim _{n \rightarrow \infty} \int_{0}^{n} A e^{-\lambda s} T(s) x d s=\lim _{n \rightarrow \infty} A \int_{0}^{n} e^{-\lambda s} T(s) x d s .
$$

Mais ainda, como

$$
\lim _{n \rightarrow \infty} \int_{0}^{n} e^{-\lambda \lambda s} T(s) x d s=\int_{0}^{\infty} e^{-\lambda \cdot s} T(s) x d s
$$

e A é fechado, concluímos de (1.5) e (1.6) que $\int_{0}^{\infty} e^{\lambda s} T(s) x d s \in D(A)$ e que

$$
A \int_{0}^{\infty} e^{-\lambda s} T(s) x d s=\int_{0}^{\infty} e^{-\lambda s} T(s) A x d s
$$

A prova está completia.

Esstamos agora em condições de mostrar o clássico Teorema de Hille-Yosida, o qual estabelece condições necessárias e suficicntes para que um operador $A$ seja o gerador infinitesimal de um $C_{0}$-senigrupo de contraçōes. 
Teorema 1.16. (Hille-Yosida) Um operador linear $A: D(A) \subset X \rightarrow X$ é o gerador infinitesimal de um $C_{0}$-semigrapo de contraçoses $(T(t))_{t \geq 0}$ em $X$ se, e somente se:

(a) A é fechado e $\overline{D(A)}=X$;

(b) $\rho(A) \supseteq(0, \infty)$ e. $\|R(\lambda: A)\| \leq \frac{1}{\lambda}$ para todo $\lambda>0$.

Demonstração. Suponha que $A$ é o gerador infinitesimal de um $C_{0}^{\gamma}$-semigrupo de contraçóes $(T(t))_{t \geq 0} \mathrm{em} X$. A condição (a) é conseqüência direta do Corolário 1.7. Para mostrar (b), provamos inicialnnente que $\rho(A) \supseteq(0, \infty)$ e que $(\lambda I-A)^{1}=R(\lambda)$, onde $R(\lambda)$ é o operador linear limitado definido no Lema 1.15. Seja $\lambda>0$. Da definição de $R(\lambda)$, para $x \in X$ temos

$$
\begin{aligned}
\frac{T(h)-I}{h} R(\lambda) x & =\frac{1}{h} \int_{0}^{\infty} e^{-\lambda t} T(t+h) x d t-\frac{1}{h} \int_{0}^{\infty} e^{-\lambda t} T(t) x d t \\
& =\frac{1}{h} \int_{h}^{\infty} e^{-\lambda(s-h)} T(s) x d s-\frac{1}{h} \int_{0}^{\infty} e^{-\lambda t} T(t) x d t \\
& =e^{\lambda h} \int_{0}^{\infty} e^{\lambda s} T(s) x d s-\frac{1}{h} \int_{0}^{\infty} e^{-\lambda t} T(t) x d t-\frac{e^{\lambda h}}{h} \int_{0}^{h} e^{\lambda s} T(s) x d s \\
& =\frac{e^{\lambda h}-1}{h} \int_{0}^{\infty} e^{\lambda s} T(s) x d s-\frac{e^{\lambda h}}{h} \int_{0}^{h} e^{\lambda s} T(s) x d s
\end{aligned}
$$

o que, pela continuidade da funçào $t \rightarrow T(t) x$, implica que

$$
\lim _{h \rightarrow 0} \frac{T(h)-I}{h} R(\lambda) x=\lambda R(\lambda) x-x
$$

Isso mostra que $R(\lambda) x \in D(A)$ c que

$$
A R(\lambda):=\lambda R(\lambda) x \quad x, \quad \text { para todo } x \in X
$$

Por outro lado, do Lema 1.15 sabemos quc, para $x \in D(A), R(\lambda) A x=A R(\lambda) x$, o quo juntamente $\operatorname{com}(1.7)$ mostra que $R(\lambda)(\lambda I-A) x=I$ para todo $x \in D(A)$ Logo $R(\lambda)=(\lambda I \cdot A)^{-1}$ e portanto $\lambda \in \rho(A)$ se $\lambda>0$. Como $(T(t))_{t \geq 0}$ é uniformemente limitado, um simples cálculo mostra que $\left\|(\lambda I-A)^{-1}\right\|=\|R(\lambda: A)\| \leq \frac{1}{\lambda}$ para todo $\lambda>0$.

Suponha agora que $A$ é um operador linear satisfazendo (a) e (b). Se $x \in D(A)$, pelos itens (b) dos Lemas 1.10 e 1.12 concluímos que $e^{t A_{\lambda}} x$ converge uniformemente para $t$ em compactos de $[0, \infty)$ a um vetor $\tilde{T}(t) x \in X$, quando $\lambda \rightarrow \infty$. Como cada $e^{t \lambda_{\lambda}} x$ é um operador contínuo com $\left\|e^{t A_{\lambda}} x\right\| \leq 1$, segue que $T(t) \in L(D(A), X)$ e que $\|T(t)\|_{L(D(A), X)} \leq 1$, onde $D(A)$ é munido da norma induzida por $X$. Mais ainda, a família $(\tilde{T}(t))_{t \geq 0}$ é um semigrupo fortemente contínuo em $D(A)$. Como as propricdades de semigrupo são obviamente satisfeitas, somente mostramos

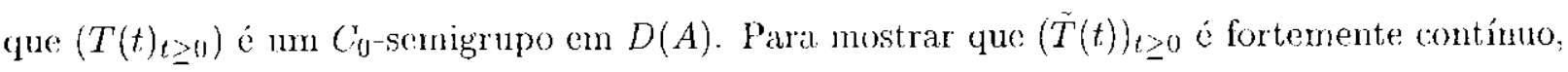


fixemos $t, h \geq 0, x \in D(A)$ e $\epsilon>0$. Como a convergência de $e^{s A_{\lambda}} x$ a $\tilde{T}(s) x$ é uniforme para $s$ em compactos de $[0, \infty)$, existem $\delta>0 \circ N_{\epsilon} \in \mathbb{N}$ tais que

$$
\left\|e^{s / \lambda} x-\tilde{T}(s) x\right\|<\frac{\epsilon}{3}
$$

se $|t-s| \leq \delta$ e $\lambda \geq N_{c}$. Usando que $\left(e^{t A_{\lambda}}\right)_{s} \geq 0$ é um semigrupo fortemente contínuo, fixamos $\delta_{1}>0$ tal que

$$
\left\|e^{(t+h) A_{N_{\epsilon}}}-e^{t A_{N_{\mathrm{t}}}}\right\|<\frac{\epsilon}{3}, \quad \text { se }|h|<\delta_{1}
$$

Das escolhas anteriores, para $0<h<\min \left\{\delta_{1}, \delta\right\}$ temos

$$
\begin{aligned}
\|\tilde{T}(t+h) x-\bar{T}(t) x\| \leq & \left\|\tilde{T}(t+h) x-e^{(t+h) A_{N_{+}}} x\right\|+\left\|e^{(t+h) A_{N_{\epsilon}}}-e^{t A_{N_{\epsilon}}}\right\| \\
& +\left\|e^{t A_{N_{\epsilon}}} x-\bar{T}(t) x\right\| \\
< & \epsilon,
\end{aligned}
$$

de onde concluímos que a semigrupo é fortemente contínuo.

Segue das considerações anteriores e da densidade de $D(A)$ em $X$ que para todo $t \geq 0$ existe. uma única extensão $T(t) \in L(X)$ de $\tilde{T}(t)$. Mais ainda, usando as idéias anteriores inferimos que $(T(l))_{t \geq 0}$ é um semigrupo fortemente contínuo em $X$ e que $\|T(t)\|_{L(X)} \leq 1$ para todo $t \geq 0$.

Finalmente, mostramos que $A$ é o gerador infinitesimal de $(T(t))_{t \geq 0}$. Seja $B$ o gerador de $(T(t))_{1} \geq 0$. Se $x \in D(A)$ e $t>0$, do Teorema 1.6 e das definições de $(T(t))_{t>0}$ e $A_{\lambda}$ temos

$$
\begin{aligned}
\frac{T(l) x-x}{t} & =\lim _{\lambda \rightarrow \infty}\left(\frac{e^{t A_{\lambda} x-x}}{t}\right) \\
& =\frac{1}{t} \lim _{\lambda \rightarrow \infty} A_{\lambda} \int_{0}^{t} e^{s A_{\lambda}} x d s \\
& =\frac{1}{t} \lim _{\lambda>\infty} \int_{0}^{t} e^{s \lambda_{\lambda}} A_{\lambda} x d s \\
& =\frac{1}{t} \int_{0}^{t} T(s) A x d s,
\end{aligned}
$$

o que implica que $x \in D(B)$ e $B x=A x$. Por outro lado, como $B$ é o gerador de $(T(t))_{t \geq 0}$, sabemos da primeira parte da demonstração que $1 \in \rho(B)$. Mas como $1 \in \rho(A)$ e $A=B$ em $D(A)$, temos que $(I-B) D(A)=(I-A) D(A)=X$, o que implica que $D(B)=(I-B)^{-1} X=$ $D(A)$ e, portanto, $A=B$. 


\subsection{O problema de valor inicial homogêneo}

O problema de Cauchy abstrato para $A: D(A) \rightarrow X$ consiste em cricoutrar un soluçäo para o problema de valor inicial

$$
\begin{array}{rlrl}
\dot{u}(t) & =A u(t), & & t>0, \\
\left.u(0)=u_{0}\right) & & u_{0} \in X .
\end{array}
$$

Como em geral $D(A) \neq X$, o conceito de solução de (1.8)-(1.9) merece atenção especial. Por isso, consideramos a seguinte definição:

Definição 1.17. Una função $u:[0, \infty) \rightarrow X$ é uma soluçào clássica do problema de Cauchy (1.8)-(1.9) se $u(\cdot)$ é continuamente diferenciável em $(0, \infty), u(t) \in D(A)$ para todo $t>0 e$ (1.8)-(1.9) säo satisfeitas.

Se $u_{0} \in D(A)$ e $A$ é o gerador infinitesimal de um $C_{0}$-semigrupo $(T(t))_{t \geq 0}$ : então a funçào $u(t)=T(t) u_{0}$ é uma soluçäo do problema de Cauchy (1.8)-(1.9), veja Teorema 1.6 (c). Mais ainda, $u(\cdot)$ é a única solução. De fato, se $v(\cdot)$ é outra solução. então para $t>0$ e $s \in(0, t)$ temos

$$
\begin{aligned}
\frac{d}{d t} T(s-t) v(t)= & -A T(s-t) v(t)+T(s-t) A v(t) \\
& \cdots-A T(s \quad t) v(t)+A T(s-t) v(t)=0,
\end{aligned}
$$

de onde concluímos que $T(s-t) v(t)$ é constante e portanto $T(t) x_{0}=v(t)$. Temos então 0 seguinte resultado:

Proposição 1.18. Seja $\left.u_{0} \in I\right)(A)$ e suponha que $A$ é o gerador infinitesimal de um semigrupo fortemente contínuo de operadores lineares em $X$. Então a função $u(t)=T(t) u_{0}$ é a única solução do problema de Cauchy (1.8)-(1.9).

Antes de mostrar o próximo teorema mostramos o seguinte lerra:

Lema 1.19. Seja $A: D(A) \subset X \rightarrow X$ um operador linear com $\rho(A) \neq \emptyset$. Entäo as seguinles propriedades säo válidas:

(a) A é fecthado;

(b) Se $D(A)$ é denso em $X$ entäo $D\left(A^{2}\right)$ também é denso em $X$. 
Demonstraçāo. Scjam $\lambda \in \rho(A)$ e $\left(x_{n}\right)_{n c N}$ uma sequència em $D(A)$ tal que $x_{n} \rightarrow x$ e $A x_{n} \rightarrow y$ quando $n \rightarrow \infty$. Como $\lambda(\lambda I \cdots A)^{\cdots}{ }^{1} x_{n}-(\lambda I-A)^{-1} A x_{n}=x_{n}$, da continuidade de $(\lambda I-A)^{-1}$ segue que $(\lambda I-A)^{-1}(\lambda x-y)=x$, o que implica que $x \in D(A)$ e $A x=y$. Portanto $A$ é fechado.

Mostramos agora (b). Se $\lambda \in \rho(A)$ entäo $(\lambda I \cdots A)^{-1} \overline{D(A)} \subset \overline{\left.(\lambda I-A)^{-1}(D)(A)\right)} \subset \overline{D\left(A^{2}\right)} \mathrm{e}$ portanto $D(A) \subset \overline{D\left(A^{2}\right)}$, o que mostra o resultado.

O próximo tcorema estabelece a relação entre a existéncia de soluçôes do problema de Cauchy abstrato e o fato de $A$ ser o gerador de um $C_{0}$-semigrupo.

Teorema 1.20. Seja $A: D(A) \subset X \rightarrow X$ um operador linear com $\overline{D(A)}=X$ e $\rho(A) \neq \emptyset$. Se para todo $x_{0} \in D(A)$ existe uma única solução $u\left(\cdot, x_{0}\right)$ do problema $(1.8)-(1.9)$, entấo $A$ éo gerador infinitesimal de um semigrupo fortemente contínuo em $X$.

Demonstração. Sabemos que $D(A)$ munido da norma do gráfico, $\left([D(A)]\right.$ : $\left.\|\cdot\|_{[D(A)}\right)$, ć um espaço de Banach, pois $A$ é fechado, veja Lema 1.19. Sobre o espaço $X_{1}=C\left([0,1]:[I)(A)_{i}\right)$ munido da norma da convergência uniforme definimos o operador $S:[D(A)] \rightarrow X_{1}$, onde $S x(t)=u(t, x)$. Afirmamos que $S$ é um operador linear, fechado e portanto contínuo em $X_{1}$. A linearidade é uma conseqüencia óbvia da unicidade de soluções do problema (1.8)-(1.9). Mostremos agora que $S$ é fechado. Sejaun $\left(x_{n}\right)_{n \in \mathbb{N}}$ uma seqüência en $[D(A)], x \in D(A)$ e $v \in S$ tais que $x_{n}>x$ erm $\left[D(A)_{1}^{1}\right.$ e $S x_{n} \rightarrow v$ em $X_{i}$. Usando que $S\left(x_{n}\right) \rightarrow v$ em $X_{1}$ e que

$$
S x_{n}(t)=x_{n}+\int_{0}^{t} A u\left(s, x_{n}\right) d s
$$

obtemos facilmente que

$$
v(t)-x+\int_{0}^{t} A v(s) d s
$$

o que implica que $v=u(\cdot, x)$, pelia unicidade da solução. Isto prova que $S$ é fechado. Segue agora do teorema do gráfico fechado que $S$ é contínuo e, portanto, que existe $C>0$ tal que

$$
\left.|S x|_{\chi_{1}}=\sup _{0 \leq t \leq 1}|u(t, x)|_{[D(A)]} \leq C|x|_{1} D(A)\right]
$$

para todo $x \in[D(A)]$. Depois dessas consideraçöes, é claro que, para cada $t \in[0,1]$; a funçăo $T^{\prime}(t): D(A) \rightarrow D(A)$ definida por $T(t) x=S x(t)$ é linear, contínua e $|T(t) x|_{[D(A)]} \leq C|x|_{[D(A)}$. Usando agora as idéias da demonstração do Tcorema 1.4 podemos definir $T(t)$ para $t>1$ empregando a relação $T(t) x=T(t-n) T(1)^{n}$. E da mesma demonstração deduzimos que existem $\omega>0$ e $M>0$ tais que

$$
|T(t) x|_{[D(A)]} \leq M e^{\omega t} ;\left.x\right|_{[D(A)]:} \quad x \in D(A) .
$$


Com o objctivo de obter uma extensão de cada operador $T(t)$ no espaço $X$, mostramos agora que

$$
T(t) A y=A T(t) y
$$

para todo $y \in D\left(A^{2}\right)$. Scja $y \in D\left(A^{2}\right)$ e definamos a função $v:[0, \infty) \rightarrow X$ por

$$
v(t)=y+\int_{0}^{t} u(s: A y) d s
$$

Usando que $y \in D\left(A^{2}\right)$ (neste caso $u(\cdot, A y)$ está bem definida) e que $A$ é fechado, temos

$$
\begin{aligned}
\frac{d}{d l} v(t)=u(t . A y) & =A y+\int_{0}^{t} \frac{d}{d s} u(s, A y) d s \\
& =A y+\int_{0}^{l} A u(s, A y) d s \\
& =A\left(y+\int_{0}^{t} u(s, A y) d s\right) \\
& -A v(t)
\end{aligned}
$$

e assim

$$
\frac{d}{d t} v(t)=A v(t)
$$

Como $v(0)-y$, da unicidado de solução do problema (1.8)-(1.9) concluímos que $v(t)=u(t, y)$, o que por sua vez implica que $A T(t) y=A u(t, y)=A v(t)=\frac{d}{d l} v(l)=u(t, A y)=T(l) A y$ e isso prova (1.14).

Sejam $\lambda \in \rho(A), \lambda \neq 0, y \in D\left(A^{2}\right)$ e $x=(\lambda I-A) y$. Como $y \in D(A)$, de (1.13) e (1.14) obtemos que

$$
\|T(t) x\|=-\left\|\left(\lambda_{0} I-A\right) T(t) y\right\| \leq C|T(t) y|_{[D(A)} \leq_{2} \leq C_{1} e^{\omega t}|y|_{[1)(A)]}
$$

Logo, pela definição da norma em $[D(A)],\left.|y:[x(A)]=\|y\|+\|A y\|=| y\right|_{[D(A)]} \leq C_{2}\|x\| \mathrm{e}$ cntäo

$$
\|T(t) x\| \leq C_{3} e^{\omega t}\|x\|,
$$

quando $T(t) x \in D\left(A^{2}\right)$. Resulta agora claro do Lema 1.19 que existe uma única extensäo contínua, que denotamos do mesmo modo, sobre $X$. Mais ainda, é óbvio que $(T(t))_{t \geq 0}$ é um semigrupo fortemente contínuo de operadores lineares em $X$.

Para finalizar a demonstração, mostramos que $A$ é o gerador de $\left(T^{\prime}(t)\right)_{t \geq 0}$. Seja $B$ o gerador infinitesimal de $\left(l^{\prime}(t)\right)_{t \geq 0}$. Se $x \in I(A)$, sabemos das hipóteses que $\left.\frac{d}{d t} T(t) x\right|_{t=0}-A x$, o que implica que $B x=A x$. Assim $D(A) \subset D(B)$ e $A=B \mathrm{~cm} D(A)$. 
Por outro lado, usando novamente (1.14) segue que para $y \in D\left(A^{2}\right)$

$$
e^{-\lambda t} A T(i) y-e^{-\lambda t} T(t) A y-e^{\lambda t} T(t) B y
$$

e entĩo

$$
\int_{0}^{\infty} e^{-\lambda l} T(t) A y d t=\int_{0}^{\infty} e^{-\lambda t} T(t) B y d t
$$

pois $A(y)=B(y)$. Usando agora que $A$ é fechado o o tema 1.15, deduzimos que para $\lambda \in$ $\mathbb{C} \bigcap \rho(A)$ com real $(\lambda)>\omega, A R(\lambda: B) y=R(\lambda: B) B y$ se $y \in D\left(A^{2}\right)$, o que pelo Lema 1.19 implica que $A R(\lambda: B) y=R(\lambda: B) B y$ para $y \in X$. Isso prova que $D(B) \subset D(A)$ e dá́ concluímos que $A=B$. Portanto $A$ é o gerador infinitesimal de $(T(t))_{l \geq 0}$ e a demonstraçào está completa. 


\section{Capítulo 2}

\section{O problema de Cauchy abstrato não homogêneo}

Neste capítulo estudamos o problema semilinear

$$
\begin{aligned}
& \dot{u}(t)=A u(t)+f(t, u(t)), \quad 0<t<T, \\
& u(0)=u_{0},
\end{aligned}
$$

onde $A: D(A) \subset X \rightarrow X$ é o gerador infinitesimal de um semigrupo fortemente continuo de opcradores lineares linitados $(T(t))_{t \geq 0}$ en $X$ e $f:[0, T] \times X \rightarrow X$ é uma funçăo apropriada.

\subsection{O problema de valor inicial não homogêneo}

Como auxílio no estudo do problema (2.1)-(2.2), nesta primeira seçäo consideramos o modelo diferencial mais simples

$$
\begin{aligned}
& \dot{u}(t)=A u(t)-f(t), \quad 0<t<T, \\
& u(0)=u_{0},
\end{aligned}
$$

onde $A: D(A) \subset X \rightarrow X$ é o gerador infinitesimal de um semigrupo fortemente contínuo de operadores lineares limitados $(T(t))_{t \geq 0}$ em $X$ e $f:[0, T] \rightarrow X$ é uma função apropriada.

Definição 2.1. Uma função $u:[0, T] \rightarrow X$ é uma solução clássica do problema (2.3)-(2.4) se $u \in C([0, T): X) \cap C^{1}((0, T): X), u(t) \in D(A)$ para todo $t \in(0, T)$ e (2.3)-(2.4) são verificadas. 
Se u(.) é uma solução dássica de $(2.3)-(2.4)$ e $f \in L^{1}([0, T]: X)$, então para $0<s<t<T$ temos

$$
\begin{aligned}
\frac{d}{d s}[T(t-s) u(s)] & =-A T(t-s) u(s)+T(t-s)(A u(s)+f(s)) \\
& =T(t-s) f(s)
\end{aligned}
$$

de onde segue que

$$
u(t)=T(l) x+\int_{0}^{t} T(t-s) f(s) d s
$$

Isso mostra o seguinte resultado:

Proposiçāo 2.2. Se $f \in L^{1}\left(\left[0, T^{\prime}\right]: X\right)$ entīo o problema (2.3)-(2.4) tem no máximo uma solução clássica. Além disso, se u( $)$ é soluçäo clássica entuão

$$
u(t)=T(t) x+\int_{0}^{t} T(t-s) f(s) d s, \quad t \in[0, T]
$$

Motivados pela Proposição 2.2 c pelo fato de que em geral $D(A) \neq X$, consideramos a seguinte definição:

Definição 2.3. Uma função $u \in C([0, T]: X)$ é uma soluçào fraca do problema (2.3)-(2.4) se

$$
u(t)=T(t) u_{0}+\int_{0}^{t} T(t-s) f(s) d s,
$$

para todo $t \in[0, T]$.

É importante observar que em geral a solução fraca de (2.3)-(2.4) não é unra solução clássica. Por cxemplo, assuma que $x \notin D(A)$ e que $T\left(t_{0}\right) x \notin D(A)$ para algum $t_{0}>0$. Considere o problema

$$
\begin{aligned}
\dot{u}(t) & =A u(l)+T(t) x, \quad t>0, \\
u(0) & =0 .
\end{aligned}
$$

A solução fraca de (2.5) é dada por

$$
u(t)=\int_{0}^{t} T(t-s) f(s) d s=t T(t) x .
$$

Como $T(t) x$ năo é diferenciável, (2.5) nầo possui solução clássica.

Nos próximos resultados estudamos condições sob as quais o problema (2.3)-(2.4) possıi solução clássica. 
Teorema 2.4. Sejam $u_{0} \in D(A)$ e $f \in L^{1}([0, T]: X) \cap C((0, T]: X)$ e definamos a função $v:[0, T] \rightarrow X$ por

$$
v(t)=\int_{0}^{t} T(t-s) f(s) d s, \quad 0 \leq t<T
$$

O problema (2.3)-(2.4) tem uma soluçăo clássica $u(\cdot)$ sobre $[0, T)$ se alguma das seguintes condiçôés é verificada:

(i) $v(t)$ é continuamente diferenciável em $(0, T)$;

(ii) $v(t) \in D(A)$ para todo $0<t<T$ e $A v(\cdot)$ é contínua em $(0, T)$.

Sé u(·) é uma soluçào clássica de (9.3) (2.4) sobre $\left[0, T^{\prime}\right)$, entäo v(·) satisfaz (i) e (ii).

Demonstração. Da definição de $v(\cdot)$ temos que para $t \in(0, T)$ e $h>0$

$$
\begin{aligned}
\frac{T(h)-I}{h} v(t)= & \frac{T(h)-I}{h} \int_{0}^{t} T(t-s) f(s) d s \\
= & \frac{1}{h} \int_{0}^{t} T(t+h-s) f(s) d s-\frac{1}{h} \int_{0}^{t} T(t-s) f(s) d s \\
= & \frac{1}{h}\left(\int_{0}^{t} T(t+h-s) f(s) d s-\int_{0}^{t} T(t-s) f(s) d s\right) \\
& -\frac{1}{h} \int_{t}^{t+h} T(t+h-s) f(s) d s .
\end{aligned}
$$

Assim

$$
\frac{T(h)-I}{h} v(t)=v \cdot \frac{v(t+h)-v(t)}{h}-\frac{1}{h} \int_{t}^{t+h} T(t+h-s) f(s) d s .
$$

Se $v(\cdot)$ é diferenciável em $(0, T)$, da continuidade de $f$ e de $(2.7)$ segue que $v(b) \in D(A)$ para cada $l \in(0, T)$ e que $A v(t)=\dot{v}(t)-f(t)$. Mais ainda, como $v(0)=0$ concluímos que $u(t)=T^{\prime}(t) u_{0}+v(t)$ é solução clássica de (2.3)-(2.4). Portanto se vale (i), o problema (2.3)-(2.4) possui una solução clássica.

Suponha agora válicla a condição (ii). Como $v(l) \in D(A)$ para todo $t \in(0, T)$, segue da continuidade de $f$ e de $(2.7)$ que $v(t)$ é diferenciável à direita em $t \in(0, T)$ e que $\frac{d^{+}}{d t} v(t)=$ $A v(t)+f(t)$. Mais ainda, $\frac{d^{+}}{d t} v(t)$ é contínua em $(0, T)$ pois $A v(\cdot)$ é contínua. Isto implica que $v(\cdot)$ é continuamente diferenciável e que $v(t)=A v(t)+f(t)$. Portanto $u(t)=T(t) u_{0}+v(t)$ é solução clássica de (2.3)-(2.4).

Se $u(\cdot)$ é solução clássica de $(2.3)$ - $(2.4)$, segue facilmente da continuidade de $f(\cdot)$ o de $(2.7)$ que as condiçôes (i) e (ii) são verificadas e por isso omitiremos os detalhes desta parte da demostração. A prova está completa. 
Corolário 2.5. Se $u_{0} \in D(A)$ e $f \in C^{1}([0, T]: X)$, cntäo o problema (2.3)-(2.4) possui uma soluçấo clássica sobre $[0, T)$.

Demonstração. Pelo Teorema 2.4 é suficicnte provar que a função $v(\cdot)$ definida om (2.6) é contimuamente diferenciável cm $(0, T)$. Sejam $l \in(0, T)$ e $h>0$ tal que $t+h \in(0, T)$. Da definição de $v(\cdot)$, temos

$$
\begin{aligned}
\frac{v(t+h)-v(t)}{h}= & \int_{0}^{t+h} T(t+h-s) f(s) d s-\frac{1}{h} \int_{0}^{t} T(t-s) f(s) d s \\
= & \frac{1}{h} \int_{0}^{h} T(t+h-s) f(s) d s+\frac{1}{h} \int_{0}^{t} T(t-s) f(s+h) d s \\
& -\frac{1}{h} \int_{0}^{t} T(t-s) f(s) d s \\
= & \frac{1}{h} \int_{0}^{h} T(t+h-s) f(s) d s+\int_{0}^{t} T(t-s)\left[\frac{f(s+h)-f(s)}{h}\right] d s
\end{aligned}
$$

e assim

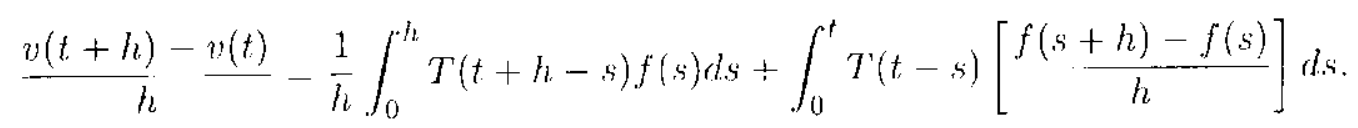

Como $f \in C^{1}([0, T]: X)$, segue de (2.8) que

$$
v^{\prime}(t)=T(t) f(0)+\int_{0}^{t} T(t-s) f^{\prime}(s) d s,
$$

para todo $t \in[0, T)$. Portanto $v(\cdot)$ é continuamente diferenciável en $(0, T)$. A prova está completia.

Corolário 2.6. Se $u_{0} \in D(A)$ c $f \in C([0, T]:[D(A)])$, entâo o problema (2.3)-(2.4) tem uma solução clássica.

Demonstração. Seja $v(\cdot)$ a função definida cm (2.6). Segue do Lema 1.14 que $v(t) \in D(A)$ e

$$
A v(t)=\int_{0}^{t} T(t-s) A f(s) d s
$$

para todo $t \in[0, T]$. Agora a propriedade é conseqüência da condição (ii) do Teorena 2.4.

Definição 2.7. Uma função $u \in C([0, T]: X)$ é uma soluçäo forte de (2.3)-(2.4) se $u^{\prime} \in L^{1}([0, T]: X), u(0)=u_{0}$ e a equaçüo (2.3) é satisfeita para quase todo $t \in[0, T]$.

É óbvio que a solução clássica de (2.3)-(2.4) é uma solução forte. Entretanto se $A \equiv 0$ c $f \in L^{1}([0, T]: X)$, mas $f \notin C([0, T]: X)$, a função $u(t)=x+\int_{0}^{l} f(s) d s, x \in X$ : ć urna solução forte de (2.3)-(2.4), mas não é clássica.

Como no caso de soluçōes clássicas, estudamos a seguir condições suficientes para que uma solução fraca scja uma solução forte. 
Teorema 2.8. Sejam $\left.u_{0} \in D(A), f \in L^{1}([0, T)]: X\right)$ e v(.) a função definida em $[0, T)$ por

$$
v(t)=\int_{0}^{t} T(t-s) f(s) d s
$$

O problema (2.3)-(9.4) tem soluçäo forte se alguma das seguintes condiçoes é satisfeita:

(i) v(t) é diferenciúvel q.t.p em $[0, T]$ e vं(t) $\in L^{1}([0, T]: X)$;

ii) $v(t) \in D(A)$ q.t.p $e m[0, T]$ e $A v(\cdot) \in L^{1}([0, T]: X)$.

Mais ainda, se u $\in C([0, T]: X)$ é uma soluçâo forte de (2.3)-(2.4) então v(.) satisfaz as condições (i) e (ii).

Demonstração. A demonstraçäo se faz usando as mesmas idéias da prova do 'leorema 2.4. Apenas observamos que como $f \subset L^{\mathrm{l}}([0, T]: X)$ o termo $\frac{1}{h} \int_{l}^{t+h} T(t+h-s) f(s) d s \mathrm{~cm}(2.7)$ converge a $f(t)$ q.t.p sobre $[0, T]$.

\subsection{O problema quase linear}

Estudamos nesta seção o problema scmilinear

$$
\begin{array}{ll}
\dot{u}(t)=A u(t)+f(t, u(t)), & \quad l \in(0, T), \\
u(0)=u_{0} \subset U \subset X, &
\end{array}
$$

onde $A$ é o gerador infinitesinal de un semigrupo fortemente contínuo de operadores lineares limitados $(T(t))_{t \geq 0}$ en $X, U \subset X$ é aberto e $f:[0, T] \times U \rightarrow X$ é uma função contínua.

Por analogia ao problema de Cauchy (2.3)-(2.4), adotamos as seguintes definiçóes:

Definição 2.9. Uma função $u \in C([0, T]: U)$ é uma soluçäo fraca do problema de Cauchy (2.9)-(2.10) se

$$
u(t)=T(t) u_{0}+\int_{0}^{t} T(t-s) f(s ; u(s)) d s
$$

para todo $t \in[0, T]$.

Definiçāo 2.10. Uma funçäo $u:\left[0, T^{\prime}\right] \rightarrow X$ é uma soluçäo clássica do problema (2.9) (2.10) se $u \in C([0, T]: X) \cap C^{1}((0, T): X), u(t) \in D(A)$ para todo $t \in(0, T)$ e $u(t)$ sutisfaz (2.9)-(2.10).

Definiçāo 2.11. Uma funçio $u \in C([0, T]: X)$ é uma soluçäo forte do problema (2.9)-(2.10) se $u^{\prime} \in L^{1}([0, T]: X), u(0)=u_{0}$ e a equaçáo (2.9) é satisfeita para quase todo $t \in\left[0, T^{1}\right]$. 
Usando o princípio de contração, apresentamos a seguir um resultado de existência e unicidade de solıção fraca para o problema (2.9)-(2.10).

Teorema 2.12. Sejam $u_{0} \in X$ e $f \in C([0, T] \times X: X)$ e suponha que existe $L>0$ tal qut.

$$
\|f(t, x)-f(t, y)\| \leq L\|x-y\|
$$

para todo $x, y \in X$. Entío existe uma única solução fraca $u\left(\cdot, u_{0}\right)$ do problema de Canchy (2.9)-(2.10). Mais ainda, a aplicação $\Gamma: X \rightarrow C([0, T]: X)$ definida por $\Gamma u_{0}=u\left(\cdot, u_{0}\right)$ é Lipschitz.

Demonstração. No espaço $C([0, T]: X)$ munido da norma da convergência uniforme, $\|\cdot\|_{T}$, definimos o operador $F: C([0, T]: X) \rightarrow C([0, T]: X)$ por

$$
F_{u}(t)=T(t) u_{0}+\int_{0}^{t} T(t \cdot s) f(s, u(s)) d s, \quad t \subset[0, T] .
$$

Mostramos a seguir que $F$ é uma contração em $C([0, T]: X)$. Seja $M=\sup _{t \in\left[0, T^{*} ;\right.}\|T(t)\|$ e fixemos $u, v \in C([0, T]: X)$. Da definição de $F$, temos que para $t \in[0, T]$

$$
\begin{aligned}
\|F u(l)-F v(t)\| & \leq M L \int_{0}^{t} \| u(s)-v(s) \mid i d s \\
& \leq M L t\|u-v\|_{T} .
\end{aligned}
$$

Similarmente,

$$
\begin{aligned}
\left\|F^{2} u(t)-F^{2} v(l)\right\| & \leq \int_{0}^{t} M\|f(s, F u(s))-f(s, F v(s))\| d s \\
& \leq \int_{0}^{t} M L\|F u(s)-F v(s)\| d s \\
& \leq \int_{0}^{t} M^{2} L^{2} s\|u-v\|_{T} d s \\
& \leq M^{2} L^{2} \frac{t^{2}}{2}\|u-v\|_{T}
\end{aligned}
$$

ou seja,

$$
\left\|F^{2} u-F^{2} v\right\|_{T} \leq M^{2} L^{2} \frac{t^{2}}{2}\|u-v\| \gamma
$$

É fácil mostrar que em geral

$$
\left\|F^{n \cdot i_{u}} u(t)-F^{n-1} v(t)\right\| \leq M^{n-1} L^{n-1} \frac{\frac{1}{n}^{n} \cdot 1}{(n-1) !}\|u-v\| T
$$

o que implica que

$$
\left\|F^{n} u-F^{n} v\right\|_{T} \leq \frac{(M L T)^{n}}{n !}\|u-v\|_{T}
$$


Como $\stackrel{(M L T)^{n}}{n !}-\cdots>0$ quando $n \rightarrow \infty$, vemos que para $n$ suficientemente grande $F^{n}$ é coutraçàn e portanto $F(\cdot)$ tem um ponto fixo $u\left(\cdot, u_{0}\right) \in C([0, T]: X)$. Glaramente este ponto fixo é uma solução fraca do problema (2.9)-(2.10).

Estudamos agora a aplicação $u_{0} \rightarrow u\left(\cdot, u_{0}\right)$. Sejam $u_{0}, v_{0} \in X$ e $u=u\left(\cdot, x_{0}\right), v=v\left(\cdot, x_{0}\right)$. Para $t \in[0, T]$ temos

$$
\begin{aligned}
\|u(t)-v(t)\| & \leq\left\|T(t) u_{0}-T^{T}(t) v_{0}\right\|+\int_{\hat{0}}^{t} \| T^{\prime}(t-s)(f(s, u(s))-f(s, v(s)) \| d s \\
& \leq M\left\|u_{0}-v_{0}\right\|+M L \int_{0}^{T}\|u(s)-v(s)\| d s,
\end{aligned}
$$

o que pela desigualdade de Gronwall-Bellman implica que

$$
\left\|u(t)-v(t): \leq M e^{M L T}\right\| u_{0} \quad v_{0} \|
$$

e portanto

$$
\|u-v\|_{T} \leq M e^{M L T}\left\|u_{0}-v_{0}\right\|
$$

Isso mostra que a aplicação $u_{0} \rightarrow u\left(\cdot, u_{0}\right)$ é Lipschitz e que a soluçăo fraca de $(2.9)-(2.10)$ é única. A prova do teorema agora está completa.

A demonstração do próximo resultado é análoga à do Teorema 2.12 e por esta razão será omitida.

Corolário 2.13. Seja $g \in C([0, T]: X)$ e suponha que se verificam as condiçóes do Teorema 2.12. Entäo a equaçâo integral

$$
w(t)=g(t)+\int_{0}^{t} T(t-s) f(s, w(s)) d s, \quad t \in[0, T],
$$

tem uma única solução $w \subset C([0, T]: X)$.

Para mostrar o próxino teorema, precisamos do seguinte lema:

Lema 2.14. Sejam $u_{0} \in D(A)$ e $f \in C([0, T] \times X: X)$ e assuma que existe $L>0$ tal que

$$
\|f(t, x)-f(s, y)\|<L(|t-s|+\|x-y\|)
$$

para todo $x, y \in X$ e todo s, $t \in[0, T]$. Entũo a soluçäo fraca do problema (2.9)-(\%.10) é Lipschitz em $[0, T]$.

Demonstração. Sejam $u(\cdot)=u\left(\cdot, u_{0}\right)$ a solução fraca de $(2.9)-(2.10), M=\sup _{t \in[0, T]}\|T(t)\|$ e $N=\sup _{t \in[0, T]}\|f(t, u(t))\|$ e fixemos $c_{1}>0$ tal que

$$
\left\|T(t+h) u_{0} \cdots T(t) u_{0}\right\|<c_{1} h
$$


para todo $t \in[0, T]$. Da definição de soluçio fraca temos

$$
\begin{aligned}
\|u(t+h)-u(t)\| \leq & \left\|T(t+h) u_{0}-T(t) u_{0}\right\|+\int_{0}^{h}\|T(t+h-s) f(s \cdot u(s))\| d s \\
& +\int_{0}^{t} \mid T(t-s)(f(s+h, u(s+h))-f(s, u(s))) \| d s \\
\leq & c_{1} h+M N h+M \int_{0}^{t} L[h+\|u(s+h)-u(s)\| d s \\
\leq & \left(c_{1}+M N+L T M\right) h+M L \int_{0}^{t}\|u(s+h)-u(s)\| d s .
\end{aligned}
$$

Assim pela desigualdade de Gronwall-Bellman

$$
\|u(t+h)-u(t)\| \leq\left(c_{1}+M N+L T M\right) e^{M L T} h
$$

o que prova o resultado.

Como no caso do problema homogêneo, uma solução fraca não necessariamente é uma solução clássica. O teorema a seguir estabclece condições sob as quais uma solução fraca ć uma solução clássica. No próximo resultado, para uma função diferenciável $f:[0, T] \times X \rightarrow X$ introduzimos a decomposição

$$
f(t+s, x+y)-f(t, x)=\left(D_{1} f(t, x), D_{2} f(t, x)\right)(s, y)+\|(s, y)\| R(t, x, s, y),
$$

onde

$$
R(t, x, s, y) \rightarrow 0, \text { se }(s, y) \rightarrow 0
$$

Teorema 2.15. Seja $u_{0} \in D(A)$ e assuma que $f(\cdot)$ verifica as condiços do Lema 2.14. Se além disso $f(\cdot)$ é continuamente diferenciável em $(0, T)$, então a solução fraca $u(\cdot)=u\left(\cdot, u_{0}\right)$ de (2.9)-(9.10) é uma solução clússica.

Demonstração. Seja $v \in C([0, T]: X)$ tal que

$$
\begin{aligned}
v(t)= & T(t) A u_{0}+T(t) f\left(0, u_{0}\right)+\int_{0}^{l} T(t-s) D_{1} f(s, u(s)) d s \\
& +\int_{0}^{l} T(l-s)\left\lfloor D_{2} f(s, u(s))\right](v(s)), d s \\
v(0)= & A u_{0}+f\left(0, u_{0}\right) .
\end{aligned}
$$

A existência e unicidade de solução para a equação integral (2.11)-(2.12) segue diretamente do Corolário 2.13, pois a função $s \mapsto D_{2} f(s, u(s)) \in C([0, T]: L(X))$. Mostramos agora que $u^{\prime}(\cdot)=v(\cdot)$. Sejann $M=\sup _{t \in[0, T]}\|T(t)\|$ e $N=\sup _{s \in[0, T]}\left\|D_{2} f(s, u(s))\right\|$. Parato $c>0$, fixemos 
$\delta>0$ tal que

$$
\begin{aligned}
\left\|\frac{T(h)-I}{h} u_{0}-A u_{0}\right\| & <\frac{\epsilon}{3 M}, \\
\left\|T(h) f(\theta, u(\theta))-f\left(0, u_{0}\right)\right\| & <\frac{\epsilon}{3 M} .
\end{aligned}
$$

Da definiçào de solução fraca, para $0<h<\delta e t \in[0, T)$ temos

$$
\begin{aligned}
& \left\|\frac{u(t+h)-u(t)}{h}-v(t)\right\| \\
& \leq\left\|I^{\prime}(t)\left(\frac{T(h) u_{0}-u_{0}}{h}-A u_{0}\right)\right\| \\
& +\frac{1}{h} \int_{0}^{h}\|T(t)[T(h-s) f(s, u(s))-f(0, u(0))]\| d s \\
& +\int_{0}^{t} M\left\|\frac{f(s+h, u(s+h))-f(s, u(s))}{h}-D_{1} f(s, u(s))-\left[D_{2} f(s, u(s))\right](v(s))\right\| d s \\
& \leq \frac{2 \epsilon}{3}+M \int_{0}^{t}\left\|\left[D_{2} f(s, u(s))\right] L(X)\right\| \frac{u(s+h)-u(s)}{h} \cdots v(s) \| d s \\
& +M \int_{0}^{t} \frac{\|h, u(s+h)-u(s)\|}{h}\|R(s \cdot u(s), h: u(s+h)-u(s))\| d s,
\end{aligned}
$$

o que pelo Lema 2.14 implica que

$$
\begin{aligned}
\left\|\frac{u(t+h)-u(t)}{h}-v(t)\right\| \leq & \begin{array}{c}
2 \epsilon \\
3
\end{array}+M N \int_{0}^{t}\left\|\frac{u(s+h)-u(s)}{h}-v(s)\right\| d s \\
& +M(1+C) \int_{0}^{t}\|R(s, u(s), h, u(s+h)-u(s))\| d s ;
\end{aligned}
$$

onde $C$ é a constante Lipschitz de $u(\cdot)$ sobre $[0, T]$. Como $R(s, u(s), h, u(s+h)-u(s))$ é uniformemente limitada para $s \in[0, T] \mathrm{e}\|R(s, u(s), h, u(s+h)-u(s))\| \rightarrow 0$ quando $h \rightarrow 0$, para cada $s \in[0, T]$, podenos reescrever a última desigualdade na forma

$$
\left\|\frac{u(t+h)-u(t)}{h}-v(t)\right\| \leq \frac{2 c}{3}+\xi(h)+M N \int_{0}^{t}\left\|\frac{u(s+h)-u(s)}{h} \cdots v(s)\right\| d s
$$

onde $\xi(h) \rightarrow 0$ se $h \rightarrow 0$. Assim por Gronwall-Bellman temos

$$
\left\|\frac{u(t+h)-u(t)}{h}-v(t)\right\| \leq\left(\frac{2 \epsilon}{3}+\xi(h)\right) e^{M N T},
$$

o que mostra que $u^{\prime}(\cdot)$ existe en $[0, T)$ c que $u^{\prime}(\cdot)=v(\cdot)$. Com estimativas similares se prova que $u^{\prime}(T)$ existe. Isso mostra que $u^{\prime} \in C([0, T]: X)$.

Para concluir a prova, somente resta mostrar que $u(t) \in D(A)$ para todo $t \in[0, T]$. Pelo item (i) do Teorema 2.4 , sabemos que o problema de valor inicial

$$
\begin{aligned}
& w^{\prime}(t)=A w(t)+f(t, u(t)), \quad t \in[0, T], \\
& w(0)=u_{0}
\end{aligned}
$$


possuri una única soluçào clássica $w(\cdot)$, pois $f(1, u(t))$ c cont immamente diferenciável sobre [0.T] e $u_{0} \in D(A)$. Claramente. $u(\cdot)=u(\cdot)$. pelo que em particular $u(t) \in D(A)$ para todo $t \in[0, T]$. l’ortanto u.(.) ć uma solıção clásica de (2.9)-(2.10). A prova está completa.

Estudanos a seguir a existência de solnçôes fracas para (2.9)-(2.10) usando o clásico Teorema de ponto fixo de Schauder, veja Martin [4!: pp 126. Introduzimos para começar a seguinte. definiçăo:

Definição 2.16. Um sernigrupo fortemente contínuo de operadores lineares limitados $(T(t))_{t} \geq 0$ em $X$ é compacto para $t_{0}>0$ se $T(t)$ é compacto para todo $t>t_{0}$. Dizemos que $(T(t)) t \geq 0$ é compacto se é compacto para $t>0$.

Note que se $T(t)$ é compacto para $t \geq 0$ então, em particular, o operador identidade é compacto, o que somente é possível se $X$ é de dimensão finita. Como este caso é de pouco interesse, assumiremos que $T(t)$ é compacto para $t>0$.

Para mostrar nosso próximo resultado precisamos do seguinte lema:

Lema 2.17. Sejam $(T(t))_{t \geq 0}$ um semigrupo fortemente contínuo de operadores lineares limitados em $X$ e KCX relativamente compacto. Entäo o conjunto de funçöes $U=\{T(\cdot) x: x \in K\}$ é relalivamente compacto em $C\left(\left[0, T^{:}: X\right)\right.$.

Demonstração. Para mostrar o resultado, usamos o Teorema de Áscoli-Arzclá. É claro que para cada $t \in[0, T]$ o conjunto $\{T(t) x: x \in X\}$ é relativamente compacto em $X$, pois $T(l)$ é contínuo. Mostramos agora que o conjunto de funções $\{T(\cdot) x: x \in K\}$ é equicontínuo cm $t=0$. So a propriedade é falsa, entäo existem $c>0$ e seqüincias $\left(x_{n}\right)_{n \in \mathbb{N}}$ em $X$ e $\left(h_{n}\right)_{n \in \mathbb{N}}$ cm $\mathbb{R}$, com $0<h_{n} \leq \frac{1}{n}$, tais que

$$
\left\|T\left(h_{n_{1}}\right) x_{n}-x_{n}\right\| \geq \epsilon
$$

para todo $n \in \mathbb{N}$. Usando a compacidade de $K$, sabemos que existe uma subseqüencia $\left(x_{n_{j}}\right)_{n_{j} \in \mathbb{N}}$ de $\left(x_{n}\right)_{n \in \mathbb{N}}$ convergente a um ponto $x \in K$. Nessas condições temos

$$
\epsilon \leq\left\|T\left(h_{n_{j}}\right) x_{n_{j}}-x_{n_{j}}\right\| \leq\left\|T\left(h_{n_{j}}\right)\right\|\left\|x_{n_{j}} \cdots x\right\|+\left\|T\left(h_{n_{j}}\right) x-x\right\|+\left\|x-x_{n_{j}}\right\|
$$

o que é absurdo, pois o lado direito da designaldade (2.13) converge a zero se $n_{j} \rightarrow \infty$, pois $(T(t))_{t \geq 0}$ é uniformemente limitado em $[0,1]$. Portanto o conjunto $U$ é equicontínuo om $t=0$. Usando agora as propriedades do senigrupo $(T(t))_{t>0}$ é fácil conchur que $\{T(\cdot) x: x \in K\}$, equicontínuo para todo $t>0$. Portanto, por Áscoli-Arzclá $\{T(\cdot) x: x \in K\}$ é relativamente compacto. 
Teorema 2.18. Sejam $U \subset X$ aberto, $u_{0} \in U$ ef $f \in C\left(\left[\left(0, I^{\prime}\right] \times U: X\right)\right.$ e assuma que o semigrupo $(T(t))_{t \geq 0}$ é compacto. Entäo existe uma soluçào fraca de (2.9)-(2.10) definida em $\left[0, t_{1}\right]$ para algum $0<t_{1} \leq T$.

Demonstração. Seja $M>0$ tal que $\|T(t)\| \leq M$ para todo $t \in[0, T]$. Usando a continuidade de $f(\cdot)$, fixamos números positivos $\delta, N>0$ tais que $B_{\delta}\left(u_{0}, X\right) \subset U$ e $\|f(t, x)\| \leq N$ para todo $(t, x) \in[0, \delta] \times B_{\delta}\left(u_{0}, X\right)$. Fixcmos agora $0<\dot{\delta}_{1}<\delta$ tal que

$$
\begin{aligned}
\| T(t) u_{0}-u_{0}: ! & <\frac{\delta}{2}, \quad 0<t<\delta_{1}, \\
M N \delta_{1} & <\frac{\delta}{2} .
\end{aligned}
$$

Sobre o espaço $P=\left\{u \in C\left(\left[0, \delta_{1}\right]: X\right): u(0)=u_{0},\left\|u(t)-u_{0}\right\| \leq \delta_{1}, \forall t \in\left[0, \delta_{1}\right]\right\}$ : definimos o operador $F: P \rightarrow C\left(\left[0, \delta_{1}\right]: X\right)$ por

$$
F u(t)=T(t) u_{0}+\int_{0}^{t} T(t-s) f(s, u(s)) d s, \quad t \in\left[0, \delta_{1}\right] .
$$

Se $F$ tem um ponto fixo, então o problema $(2.9)-(2.10)$ tem uma solução fraca em $C\left(\left[0, \delta_{1}\right]: X\right)$. Para mostrar que $F$ tem um ponto fixo em $P$, usamos o clássico Teorema de ponto fixo de Schauder, veja [4], pp 126. Por isso, a seguir mostramos que $F$ é um operador contínuo com valores em $F(P)$, que $F(P)=\{F u ; u \in P\}$ é equicontínuo em $\left[0, \delta_{1}\right]$ e que o conjunto $F P(t)=$ $\{F u(t) ; u \in P\}$ é relativamente compacto $\mathrm{cm} X$ para todo $t \in\left[0, \delta_{1}\right]$. Estudamos cada uma dessas propriedades de forma separada.

(a) A função $F$ í contínua e $F^{\top}\left(I^{\prime}\right) \subset P$.

Sejam $\left(u_{n}\right)_{n \in \mathbb{N}}$ uma seqüencia con valores em $P$ e $u \in P$ tais que $u_{n} \rightarrow u$ enr $P$. Como $f$ é limitada em $\left[0, \delta_{1}\right] \times B_{\delta_{1}}\left(u_{0}, X\right)$, segue diretamente do Teorcma da convergência dominada de Lebesgue que

$$
\int_{0}^{\delta_{1}}\left\|f\left(s, u_{n}(s)\right)-f(s, u(s))\right\| d s \rightarrow 0
$$

se $n \rightarrow \infty$, o que implica a continuidade de $F$. Por outro lado, se $u \in P$ e $t \in\left[0, \delta_{1}\right]$, da definição de $F$ e de (2.14), (2.15) temos

$$
\begin{aligned}
\left\|F u(t)-u_{0}\right\| & \leq\left\|T(t) u_{0}-u_{0}\right\| \div\left\|\int_{0}^{t} T(t-s) f(s, u(s))\right\| d s \\
& <\frac{\delta}{2}+M N \delta_{1} \\
& <\delta
\end{aligned}
$$

o que mostra que $F^{r} u \in P$ e portanto $F(P) \subset P$. 
(b) $F P(t)$ é relativamente compacto cm $X$ para todo $t \in\left[0, \delta_{1}\right]$.

Se $t=0$ a propriedade é óbvia. Sejam $t>0$ e $0<t<1$. Se $u \in I$, da definição de $F$ vemos que

$$
\begin{aligned}
F u(t) & =T(t) u_{0}+\int_{0}^{t-\epsilon} T(t-s) f(s, u(s)) d s+\int_{t-\epsilon}^{t} T(t-s) f(s, u(s)) d s \\
& =T(t) u_{0}+T(\epsilon) \int_{0}^{t-\epsilon} T(t-s-c) f(s, u(s)) d s+\int_{t, \epsilon}^{t} T(t-s) f(s, u(s)) d s .
\end{aligned}
$$

Como

$$
\begin{aligned}
\|T(t-s-\epsilon) f(s \cdot u(s))\| & \leq M N \\
\int_{t-c}^{t}\|T(t-s) f(s, u(s))\| & \leq M N c
\end{aligned}
$$

para $s \in\left[0, \delta_{1}\right]$, segue de $(2.16)$ que

$$
F u(t) \in\left\{T(t) u_{0}\right\}+T(\epsilon)\left(B_{M N \delta_{1}}(0, X)\right)+C_{c}
$$

onde $C_{\epsilon} \subset X$ e diam $\left(C_{\epsilon}\right) \leq 2 M N \epsilon$. O antcrior implica que $F P(t)$ é um conjunto totalmente limitado em $X$, pois $T(\epsilon)$ é compacto, e conseqüentemente $F P(t)$ é relativamente compacto em $X$, uma vez que $X$ é métrico.

(c) O conjunto $F P-\{F u: u \in P\}$ é cquicontínuo emr $\left[0, \delta_{1}\right]$.

Sejarn $t^{\prime} \in\left[0, \delta_{1}\right]: 0<\epsilon<t^{\prime}$ e $\epsilon^{\prime}=\frac{\epsilon}{8 M N}$. Usando que $T\left(\epsilon^{\prime}\right) B_{N}(0, X)$ é compacto, pelo Lema 2.17 fixamos $0<\varrho<\delta_{1}$ tal que

$$
\begin{aligned}
\varrho M N & <\frac{\epsilon}{4}, \\
\left\|T(h) x_{0}-x_{0}\right\| & <\frac{\epsilon}{4 M}, \quad 0<h<\varrho \\
\left\|\left(T(s)-T\left(s^{\prime}\right)\right) x\right\| & <\frac{\epsilon}{4 M},
\end{aligned}
$$

quando $x \in T\left(\epsilon^{\prime}\right) B_{N}(0, X)$ e $s, s^{\prime} \in[0, T]$ sào tais que $\left|s-s^{\prime}\right| \leq \varrho$. Nestas condiçōes, para $0<t^{\prime}<t \leq \delta_{1}, \operatorname{com} t-t^{\prime} \leq \varrho$, temos

$$
\begin{aligned}
\left\|F u\left(t^{\prime}\right)-F u(t)\right\| \leq & \left\|T\left(t^{\prime}\right)\right\|\left\|u_{0}-T\left(t \cdots t^{\prime}\right) u_{0}\right\| \\
& +\int_{0}^{t^{\prime}-\epsilon^{\prime}} M\left\|T\left(t^{\prime}-s-\epsilon^{\prime}\right)-T^{\prime}\left(t-s-\epsilon^{\prime}\right) T^{\prime}\left(\epsilon^{\prime}\right) f(s ; u(s))\right\| d s \\
& +\int_{t^{\prime} t^{\prime}}^{t^{\prime}} 2 M N d s+\int_{t^{\prime}}^{t} M N d s \\
< & M \frac{\epsilon}{1 M}+M \frac{\epsilon}{4 M}+2 M N \epsilon^{\prime}+M N \varrho, \\
< & \epsilon,
\end{aligned}
$$


de onde conclúnos que

$$
\left\|F u\left(t^{\prime}\right)-F u(t)\right\|<\epsilon,
$$

para todo $u \in P$ e todo $0<t-t^{\prime} \leq \varrho$. o que mostra que FP é equicontínuo à direita em $t$. A equicontinuidade à esquerda em $t \in\left(0, \delta_{1}{ }^{3}\right.$ é provada usando as mesmas idéias e estimativas. Portanto FB ć equicontínuo sobre $\left[0, \delta_{1}\right]$.

Segue do teorema de $\Lambda$ scoli-Arzelá e de (a), (b), (c) que $F(P)$ é rolativamente compacto om $C\left(\left[0, \delta_{1}\right]: X\right)$ e conseqüentemente $F$ ć completamente continuo. Pelo Teorema de Schauder, veja [4], pp. 126, F lem unn ponto fixo u(.) cm $P$. Obviamente u(.) é uma solução fraca de (2.9)-(2.10), o que completa a prova. 


\section{Capítulo 3}

\section{Um problema de evolução com}

\section{impulsos}

Neste capítulo estabolecemos novos resultados de existéncia de soluçóes fracas para umat classe de equaçöes funcionais impulsivas modeladas na forma

$$
\begin{aligned}
\dot{u}(t) & =A u(t)+f(t, u(t), u(a(t))), \quad t \in I=\left(0, T^{\prime}\right], \\
u(0) & =u_{0}, \\
\Delta u\left(t_{i}\right) & =I_{i}\left(u\left(t_{i}\right)\right),
\end{aligned}
$$

onde $A$ é o gerador infinitesimal de um semigrupo fortemente contínuo de operadores lineares limitados $(T(t))_{t \geq 0}$ definido sobre um espaco de Bandeh $X, 0<t_{1}<t_{2}<\ldots<t_{n}<T$, $f: I \times X^{2} \mapsto X, I_{i}: X \mapsto X$ são funções apropriadas e a notação $\Delta u\left(t_{i}\right)$ é definida por $\Delta u\left(t_{i}\right)=u\left(t_{i}^{+}\right)-u\left(t_{i}^{-}\right)$.

O problema diferencial (3.1)-(3.3) foi estudado recentemente por Liu em [3], para o caso $a(t)=t, t \in[0, T]$. Nesse trabalho, Liu mostra a existência de soluções fracas e clássicas para (3.1)-(3.3) usando o teorema de ponto fixo para contrações e o T'eorema 6.1.5 en Pazy [5], veja pp.187, a respeito de regularidade de soluçöes fracas.

Neste capítulo apresentanos novos resultados de existência de soluçóes fracas, tanto locais como globais, para o problema (3.1)-(3.3). A diferença com o trabalho de Liu [3] é que nossos resultados são provados usando critćrios de ponto fixo para operadores condensantes, especificamente Leray-Schauder e Sadovskii, veja Teoremas 3.1 e 3.2. 


\subsection{Existência de soluções fracas}

Apresentamos a seguir alguns tcoremas clássicos de ponto fixo que usamos para obter nossos resultiados.

Teorema 3.1. (Leray - Schauder) Seja $D \subset X$ convexo tal que $0 \in D$. Se $F: D \rightarrow D$ é uma funçäo completamente contínua, entäo $F$ possui um ponto fixo em $D$ ou o conjunto $\{x \in D: x=\lambda F(x), 0<\lambda<1\}$ é näo limitado em $X$.

Teorema 3.2. (Sadovskii) Seja $D \subset X$ convexo. Se $F: D \rightarrow D$ é uma função condensante, entäo $F$ tem um ponto fixo $\mathrm{em} D$.

No que se segue, $\mathcal{P} C=\mathcal{P C}([0, T]: X)$ será o espaço

$$
\mathcal{P} C=\mathcal{P} C([0, T]: X)=\left\{\begin{array}{lc}
u:[0, T] \rightarrow X: & u \in C\left(\left[0, t_{1}\right]: X\right) \cap C\left(\left(t_{i}, t_{i+1}\right]: X\right) \\
& \text { e } u\left(t_{i}\right) \text { existe, } \forall i=1, \ldots n-1
\end{array}\right\},
$$

munido da norma da convergencia uniforme $\|\cdot\|_{T}$.

Para $u \in P C([0, T]: X)$, usaremos a notação ũ para a funçâo defiricla por:

$$
\tilde{u}(t)=\left\{\begin{aligned}
u(t), & t \neq t_{i} \\
u\left(t_{i}^{-}\right), & t=t_{i}
\end{aligned}\right.
$$

e para $B \subset \mathcal{P C}([0, T]: X), \tilde{B}$ denotará o conjunto $\hat{B}=\{\tilde{u}: u \in B\}$.

Como o domínio do operador $A$ pode ser diferente de $X$, é natural pensar em um conceito mais fraco de solução para o problema (3.1)-(3.3). Se $u \in \mathcal{P} C([0, T]: X)$ é solução do problema (3.1)-(3.3), é fácil mostrar que para $t \in[0, T]$

$$
u(t)=T(t) u_{0}+\int_{0}^{l} T(t-s) f(s, u(s), u(a(s))) d s+\sum_{t_{i}<t} T\left(t-t_{i}\right) I_{i}\left(u\left(t_{i}\right)\right) .
$$

Isto motiva a seguinte definição:

Definição 3.3. Uma função $u \in \mathcal{P} C([0, T]: X)$ é uma solução fraca do problema impulsivo $(3.1)-(3.3)$ se

$$
u(t)=T(t) u_{0}+\int_{0}^{t} T(t \cdots s) f(s, u(s), u(a(s))) d s+\sum_{t_{i}<t} T\left(t-t_{i}\right) I_{i}\left(u\left(t_{i}\right)\right)
$$

para todo $t \in[0, T]$. 
Como estamos interessados em mostrar existência de soluçôes fracas para (3.1)-(3.3) usando teoremas de ponto fixo para operadores condensantes, consideramos o seguinte resultado do tipo Áscoli-Arzelá em $\mathcal{P C}([0, T]: X)$. No próximo resultado, para $u \in B$ e $i \in\{1,2, \ldots, n-1\}$ definimos as funçòes $\left.\hat{u}_{0} \in C\left(0, t_{1}\right]: X\right)$ e $\bar{u}_{i} \in C\left(\left[t_{i}, t_{i+1}\right]: X\right)$ por

$$
\begin{aligned}
& \widetilde{u}_{0}(t)=u(t) \quad \text { para } t \in\left[0, t_{1}\right], \\
& \tilde{u}_{u}(t)= \begin{cases}u(t), & \text { para } t \in\left(t_{i}, t_{i+1}\right], \\
u\left(t_{i}^{\prime}\right), & \text { para } t=t_{i} .\end{cases}
\end{aligned}
$$

Mais ainda, $\widetilde{B}_{i}, i=0,1,2, \ldots n-1$, será o conjunto $\widetilde{B}_{i}=\left\{\tilde{u}_{i}: u \in B\right\}$.

Teorema 3.4. Un conjunto $B \subset \mathcal{P} C([0, T]: X)$ é relativamente compracto se, e somente se, as seguintes propriedades se verificam:

(a) Para lodo $t \in I$, os conjuntos $B(t)=\{u(t): u \in B\}$ e $\tilde{B}(t)=\{\tilde{u}(t): u \in B\}$ são relativamente compactos em $X$;

(b) O conjunto $B$ é equicontínuo à esquerda em cada $t \in I$;

(c) O conjunto $\tilde{B}$ é equicontínuo à direita em cada $t \in I$.

Demonstração. Suponha que as condições (a). (b), (c) são verificadas. É conscqüència direta das hipóteses e do clássico Tcorema de Áscoli-Arzelá que os conjuntos $\tilde{B}_{0}$ e $\widetilde{B}_{i}, i=1,2, \ldots n$, são relativarnente compactos em $C\left(\left[0, t_{1}\right]: X\right)$ e $C\left(\left[t_{i}, t_{i+1}\right]: X\right)$, respectivamente. Seja $\left(u^{k}\right)_{k \in \mathbb{N}}$ uma seqüência em $B$. Destas considerações, existe uma subseqüiencia de $\left(u^{k}\right)_{k \in \mathbb{N}}$, que denotamos $\left(u^{k_{1}}\right)_{k_{1} \in \mathbb{N}}$, tal que $\left(u_{1}^{k_{1}}\right)_{k_{1} \in \mathbb{N}}$ é convergente a una função $v_{1} \in C\left(\left[0, t_{1}\right]: X\right)$. Similarmente, a seqüencia $\left(u^{k_{1}}\right)_{k_{1} \in N}$ possin mma subseqüencia, que denotamos $\left(u^{k_{2}}\right)_{k_{2} \in \mathbb{N}}$, tal que $\left(\tilde{u}_{2}^{k_{2}}\right)_{k_{2} \in \mathbb{F}}$ converge a uma funça $\left.v_{2} \in C\left(\mid t_{1}, t_{2}\right]: X\right)$. Continuando com esse raciocínio, conchúnos que: existe uma subseqüencia de $\left(u^{k_{n-1}}\right)_{k_{n-1} \in \mathbb{R}}$ : que denotamos $\left(u^{k_{n}}\right)_{k_{n} \in \mathbb{N}}$, tal que $\left(\tilde{u}_{n}^{k_{n}}\right)_{k_{n} \in \mathbb{N}}$ converge a uma função $v_{n} \in C\left(\left[t_{n-1}, t_{n}\right]: X\right)$. É claro quc a subseqüência $\left(u^{k_{n}}\right)_{k_{n} \in \mathbb{N}}$ converge a $u \in \mathcal{P} C$, onde $u$ é a função tal que $\tilde{u}_{i}=v_{i}$ para cada $i=1,2 \ldots, n$. lsso prova que $B$ é relativamente compacto em $\mathcal{P} C$.

Suponha agora que $B \subset \mathcal{P C}$ é relativamente compacto. Por Áscoli-Arzelá vemos facilnente que, para $t \neq t_{i}, B(t)$ e $\hat{B}(t)$ são relativamente compactos e $B$ é equicontínuo en $t$. Para cstudas o caso $t=t_{i}$, mostramos inicialmente que para cada $i=1,2, \ldots n$, a função $\psi_{i}: \mathcal{P} C \rightarrow X$ onde? $\psi_{i}(u)=u\left(t_{t}^{\dagger}\right)$ c contínua. Sejam $\epsilon>0$ e $u \in \mathcal{P} C$. Para todo $v \in B_{\frac{\varsigma}{3}}(u, \mathcal{P} C)$ existe $\delta_{v}>0$ tal 
que, $\left\|v\left(t_{i}^{+}\right)-v\left(t_{i}+h\right)\right\|<\frac{c}{3}$ quando $0<h \leq \delta_{i}$. Com estas escollas, para $v \in B_{\frac{\epsilon}{3}}(u, \mathcal{P} C)$ c $0<h \leq \delta=\min \left\{\delta_{u}, \delta_{v}\right\}$ temos

$$
\begin{aligned}
\left\|u\left(t_{i}^{+}\right)-v\left(t_{i}^{+}\right)\right\| \leq & \left\|u\left(t_{i}^{+}\right)-u\left(t_{i}+h\right)\right\|+\left\|u\left(t_{i}+h\right)-v\left(t_{i}+h\right)\right\| \\
& +\left\|v\left(t_{i}+h\right) \cdot v\left(t_{i}^{-}\right)\right\| \\
& <\epsilon,
\end{aligned}
$$

o que prova que $\psi_{i}$ é contínua e portanto $\tilde{B}\left(t_{i}\right)$ é relativamente compacto em $X$. Mostramos agora a equicontinuidade de $\tilde{B}$ à direita em $t=t_{i}$ raciocinando por absurdo. Suponha que existem $\epsilon>0$ e seqüências $\left(u^{k}\right)_{k \in \mathbb{N}} \mathrm{em} \mathcal{P} C$ e $\left(h_{n}\right)_{n \in \mathbb{N}} \mathrm{cm} \mathbb{R}$, com $0<h_{n}<\frac{1}{n}$, tais que

$$
\left\|u^{k}\left(t_{i}^{\dagger}\right)-u^{k}\left(t_{i}+h_{n}\right)\right\| \geq \epsilon
$$

Como $B$ é relativamente compacto em $\mathcal{P} C$, existem uma subseqüência de $\left(u^{k}\right)_{k \in \mathbb{R} \text { : que }}$ continuamos denotando por $\left(u^{k}\right)_{k C N}, \propto u \in \mathcal{P} C$ tais que $u^{k} \rightarrow u \mathrm{~cm} \mathcal{P} C$ quando $k \rightarrow \infty$. Da continuidade de $\psi_{i}$ podemos fixar $N_{\epsilon} \in \mathbb{N}$ tal que

$$
\begin{aligned}
& \left\|u\left(t_{i}^{+}\right)-u^{k}\left(t_{i}^{+}\right)\right\|<\frac{\epsilon}{3}, \\
& \left\|u^{n}-u\right\|_{\mathcal{P} C}<\frac{\epsilon}{3},
\end{aligned}
$$

para todo $k \geq N_{\epsilon}$. Nestas condições, para $k \geq N_{\epsilon}$ temos

$$
\begin{aligned}
\left\|u\left(t_{i}^{+}\right)-u\left(t_{i}+h_{n}\right)\right\| \geq & -\left\|u\left(t_{i}^{+}\right)-u^{k}\left(t_{i}^{-}\right)\right\|+\left\|u^{k}\left(t_{i}^{+}\right)-u^{k}\left(t_{i}+h_{n}\right)\right\| \\
& -\left\|u^{k}\left(t_{i}+h_{n}\right)-u\left(t_{i}+h_{n}\right)\right\| \\
> & -\frac{\epsilon}{3}+\epsilon-\frac{\epsilon}{3} \\
= & \frac{\epsilon}{3},
\end{aligned}
$$

o que é um absurdo pois $u \in \mathcal{P} C$. Isso mostra que $\tilde{B}$ é equicontínuo à direita em cada $t_{i}$. A prova do teorema está agora completa.

No restante deste capítulo, assumiremos que as scguintes condições são verificadas:

$\left(\mathbf{A}_{1}\right)$ A função $a: I \rightarrow I$ é contínua e $a(t) \leq t$ para todo $t \in[0, T]$;

(A $\mathbf{A}_{2}$ ) A função $f: I \times X^{2} \rightarrow X$ verifica a seguintes condiçóes do tipo Caratheodory:

(a) $f(t, \cdot): X \rightarrow X$ é contínua para quase todo $t \in[0, T]$;

(b) $f(\cdot, x): I \mapsto X$ é integrável para todo $x \in X$; 
(c) Fxistern funçōes contínuas $m:[0, T] \rightarrow[0, \infty)$ e $W:[0, \infty) \rightarrow(0, \infty)$, com $W(\cdot)$ não decrescente, tais que

$$
\|f(t, x, y)\| \leq m(t) W(\|x\|+\|y\|)
$$

para todo $t \in I$ e todo $x, y \in X$.

Teorema 3.5. Seja $u_{0} \in X$ e suponta que as seguintes propriedades são válidas:

$\left(\mathbf{H}_{1}\right)$ Os operadores $I_{i}, i=1, \ldots, n$, sĩo completamente contínuos e limitados em $X$. Seja $N_{i}=\sup \left\{\left\|I_{i}(x)\right\|: x \in X\right\}$;

$\left(\mathbf{H}_{2}\right)$ Para todo $t \in[0, T]$ e $r>0$ o conjunto $\{T(t) f(s, x, y): s \in[0, t],\|x\| \leq r,\|y\| \leq r\}$ é relativamente compacto em $X$.

Se $2 M \int_{0}^{T} m(s) d s<\int_{c}^{\infty} \frac{d s}{W(s)}$, onde $c=2\left(M\left\|u_{0}\right\|+\sum_{i=1}^{n} M N_{i}\right)$ c $M=\sup _{t \in[0, T]}\|T(t)\|$, então o problema (3.1)-(3.3) tem pelo menos uma solução fraca.

Demonstraçāo. Seja $\Gamma: \mathcal{P} C([0, T]: X) \mapsto P C([0, T]: X)$ a furç̧ào definida por

$$
\Gamma u(t)=T(t) u_{0}+\int_{0}^{t} T(t-s) f(s, u(s), u(a(s))) d s+\sum_{t_{i}<t} T\left(t-t_{i}\right) I_{i}\left(u\left(t_{i}\right)\right), \quad t \in 1 .
$$

A função $\Gamma$ está ben definida e pelo Tenrema da convergência dominada de Lebesgue é fácil mostrar que $\Gamma$ é contínua. Como um ponto fixo de $\Gamma$ é uma solução fraca de (3.1)-(3.3), no que vem a seguir mostramos que T' verifica as hijóteses do Teorcma 3.1. Primeiro provamos que o conjunto $\{u \in \mathcal{P} C: \lambda \Gamma u=u, \lambda \in(0,1)\}$ é limitado em $\mathcal{P} C([0, T]: X)$. Seja $\lambda \in(0,1)$ e assuma que $u_{\lambda} \in \mathcal{P C}$ é tal que $\lambda \mathrm{I}^{\prime} u_{\lambda}=u_{\lambda}$. Diretamente da definição de I' temos

$$
\begin{aligned}
\left\|u_{\lambda}(t)\right\| \leq & \lambda\|T(t)\|\left\|u_{0}\right\|+\lambda \int_{0}^{t}\left\|T(t-s) f\left(s, u_{\lambda}(s), u_{\lambda}(a(s))\right)\right\| d s \\
& \left.+\lambda \sum_{t_{i}<l} \| T\left(t-t_{i}\right) I_{i}\left(u_{\lambda} t_{i}\right)\right) ! \\
\leq & M\left\|u_{0}\right\|+M \int_{0}^{i} m(s) W\left(\left\|u_{\lambda}(s)\right\|+\left\|u_{\lambda}(a(s))\right\|\right) d s+\sum_{i=1}^{n} M N_{i} .
\end{aligned}
$$

Denotando por $x_{\lambda}(t)$ o lado direito da desigualdade anterior, temos

$$
x_{\lambda}^{\prime}(t) \leq M m(t) W\left(2 x_{\lambda}(l)\right)
$$

pois $W(\cdot)$ é não decrescente e $a(t) \leq t$ para todo $t \in I$. Segue de (3.5) e da hipótese que

$$
\int_{2 w_{\lambda}(0)}^{2 x_{\lambda}(t)} \frac{d s}{W(s)} \leq 2 M \int_{0}^{T} m(s) d s<\int_{c}^{\infty} \frac{d s}{W(s)},
$$


o que nos permite concluir que o conjunto de funçies $\left\{x_{\lambda}: \lambda \in(0,1)\right\}$ é limitado em $C\left(\left[0, T^{\dagger}: X\right)\right.$ c: conscqüentemente. que o conjunto $\left\{u_{\lambda}: u_{\lambda}=\Gamma^{\prime} u_{\lambda}, \lambda \in(0,1)\right\}$ é limitado em $\mathcal{P} C([0, T]: X)$. pois $\left\|u_{\lambda}(l)\right\| \leq x_{\lambda}(t)$ para todo $t \in[0, T]$.

Mostramos agora que para todo $B \subset \mathcal{P C}$ limitado; o conjunto $\Gamma B=\{\Gamma u: u \in B\}$ ć relativamente compacto em $\mathcal{P} C$. Para isto, dado $r>0$ lixamos $B_{r}=B_{r}(0, \mathcal{P} C)$ e consideramos a decomposiçäo $\mathrm{l}^{\prime}=\Gamma_{1} \mid \Gamma_{2}$, onde

$$
\begin{aligned}
& \Gamma_{1} u(t)=T(t) u_{0}+\sum_{t_{i}<t} T\left(t-t_{i}\right) I_{i}\left(u\left(t_{i}\right)\right), \\
& \Gamma_{2} u(t)=\int_{0}^{t} T(t-s) f(s, u(s), u(a(s))) d s .
\end{aligned}
$$

Usando o Teorema 3.4 , nostramos a seguir que os conjuntos $\Gamma_{i}\left(B_{r}\right), i=1,2$, sầo relativamente, compactos em $P C$. Estudamos cada caso separadamente.

Caso $1 \mathrm{O}$ conjunto $\Gamma_{1}\left(B_{r}\right)$ é relativamente compacto em $\mathcal{P} C$.

Começatmos mostrando a equicontimuidado dos conjuntos $\Gamma_{1}\left(B_{r}\right), \widehat{\Gamma_{1}\left(B_{r}\right)}$. Sejam $\epsilon>0$, $t \in I \backslash\left\{t_{1}, \ldots, t_{n}\right\}$ e $\delta_{1}>0$ tal que $\left\{t_{1}, \ldots, t_{n}\right\} \cap\left[t \quad \delta_{1}, t+\delta_{1}\right]=\emptyset$. Como o conjunto $\bigcup_{n=1}^{n} I_{i}\left(B_{r}(0, X)\right)$ ć relativamente compacto em $X$ e $\left(T^{\prime}(t)\right)_{t \geq 0}$ é fortemente contínuo, pelo Lema 2.17 existe $0<\delta_{2}<\delta_{1}$ t.al que

$$
\begin{aligned}
\left\|(T(t)-T(s)) I_{i}(x)\right\| & <\frac{\epsilon}{2 n}, \quad x \in \bigcup_{i=1}^{n} I_{i}\left(B_{r}(0, X)\right), \\
\left\|(T(t)-T(s)) u_{0}\right\| & <\frac{\epsilon}{2},
\end{aligned}
$$

quando $|t-s| \leq \delta_{2}$. Nestas condições, para $0<h<\delta_{2}$ e $u \in B_{r}$ temos

$$
\begin{aligned}
\| \Gamma_{1} u(t & -h)-\Gamma_{1} u(t) \| \\
& \leq\left\|(T(t-h)-T(t)) u_{0}\right\|+\left\|\sum_{t_{3}<t}\left(T\left(t-h-t_{i}\right) \cdots T\left(t-t_{i}\right)\right) I_{i}\left(u\left(t_{i}\right)\right)\right\| \\
& <\frac{\epsilon}{2}+\sum_{t_{i}<t}\left\|\left(T\left(t-h-t_{i}\right) \cdots T\left(t \cdots t_{i}\right)\right) I_{i}\left(u\left(t_{i}\right)\right)\right\| \\
& <\frac{\epsilon}{2}+\sum_{i=1}^{n} \frac{\epsilon}{2 n} \\
& =\epsilon
\end{aligned}
$$

o que mostra a equicontinuidade de $\Gamma_{:} B_{r}$ à esquerda em $t \neq t$, . A equicontinuidade de $\widehat{\Gamma_{1} B_{r}}$ à direita em $t \neq t_{i}$ é provada de forma similar. 
Soja agora $t=t_{i}$ e fixemos $\delta>0$ tal que $\left\{t_{j}: j \neq i\right\} \cap\left[t_{i}-\delta, t_{i}+\delta\right]=\emptyset$ e (3.8)-(3.9) sejam satisfeitas. Da definição de $\Gamma$, para $u \in B_{r}$

$$
\Gamma_{1} u\left(t_{i}\right)=T\left(t_{i}\right) u_{0}+\sum_{j=1}^{i-1} T\left(t_{i}-t_{j}\right) I_{j}\left(u\left(l_{j}\right)\right)
$$

e cutico para $0<h<\delta$

$$
\begin{aligned}
\left\|\Gamma_{1} u\left(t_{i}-h\right)-\Gamma_{1} u\left(t_{i}\right)\right\| \leq & \left\|\left(T^{\prime}\left(t_{i}-h\right)-T\left(t_{i}\right)\right) u_{0}\right\| \\
& +\sum_{j=1}^{i-1}\left\|\left(T\left(t_{i}-h_{1}-t_{j}\right)-T\left(t_{i}-t_{j}\right)\right) I_{j}\left(u\left(t_{j}\right)\right)\right\| \\
< & \frac{\epsilon}{2}+\frac{\epsilon}{2}=\epsilon .
\end{aligned}
$$

e portanto $\Gamma_{1} B_{r}$ é equicontínuo à esquerda em $t=t_{i}$.

Mostramos agorà a equicontinuidade de $\widehat{\Gamma_{1} B_{r}}$ à dircita $\mathrm{cm} t=t_{i}$. Pela definiçăo de $\widetilde{\Gamma_{1} u}$, para $u \in B_{r}$

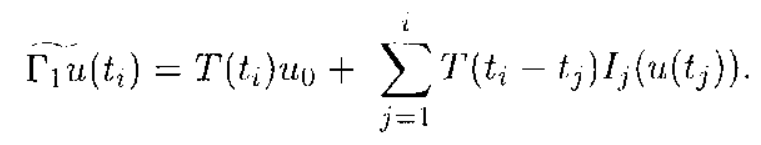

Fixemos $\delta>0$ tal que $\left\{t_{j}: j \neq i\right\} \cap\left[l_{i}-\delta, t_{i}+\delta\right]=\emptyset \mathrm{c}(3.8)$-(3.9) sejam válidas. Para $u \in B_{r}$ e $0<h<\delta$ vemos que

$$
\begin{aligned}
\left\|\widetilde{\Gamma_{1} u}\left(t_{i}+h\right)-\widehat{\Gamma_{1} u}\left(t_{i}\right)\right\| \leq & \left\|\left(T\left(t_{i}+h\right) \cdots T\left(t_{i}\right)\right) u_{0}\right\| \\
& +\left\|\sum_{j-1}^{i}\left(T\left(t_{i}+h-t_{j}\right)-T\left(t_{i}-t_{j}\right)\right) I_{j}\left(u\left(t_{j}\right)\right)\right\| \\
< & \frac{\epsilon}{2}+\frac{\epsilon}{2}=\epsilon,
\end{aligned}
$$

o que mostra $\widetilde{\Gamma_{1}} B_{r}$ é equicontínuo à direita em $t=t_{i}$.

Isso mostra que $\Gamma_{1} B_{r}$ verifica a condiçôcs (b), (c) do Teorema 3.4.

Mostramos agora que $\Gamma_{1} B_{r}(t)$ e $\widetilde{\Gamma_{1} B_{r}}(t)$ são relativamente compactos para todo $t \in[0, T]$. Como os opcradores $I_{i}$ são completamente contínuos, para cada $s \in I$ o conjunto $U(s, r)=$ $\left\{T(s) I_{i}(x):\|x\| \leq r, i=1,2, \ldots, n\right\}$ é relativamente compacto $\mathrm{em} X$. Se $t \neq t_{i}, i \in \mathbb{N}, \mathrm{e} u \in B_{r}$

$$
\begin{aligned}
\mathrm{F}_{1} B_{T}(t) & \subseteq\left\{T(t) u_{0}\right\}+\left\{\sum_{t_{i}<t} T\left(t-l_{i}\right) I_{i}(x):\|x\| \leq r\right\} \\
\subseteq & \left\{T(t) u_{0}\right\}+\sum_{t_{i}<t} U\left(t-t_{i}, r\right)
\end{aligned}
$$

o que prova que $\Gamma_{1} B_{r}(t)$ é relativamente compacto em $X$ para $t \neq t_{i}, i=1,2, \ldots, n$. 
Histudemos agora o caso $t=l_{i}$. Como

$$
\begin{aligned}
& \Gamma_{1} u\left(t_{i}\right)=T\left(t_{i}\right) u_{0}+\sum_{j-1}^{i-1} T\left(t_{i}-t_{j}\right) I_{j}\left(u\left(t_{j}\right)\right) \\
& \Gamma_{1} u\left(t_{i}\right)-T\left(t_{i}\right) u_{0}+\sum_{j=1}^{i} T\left(t_{i}-t_{j}\right) I_{j}\left(u\left(t_{j}\right)\right),
\end{aligned}
$$

vemos que para $u \subset B_{r}$

$$
\begin{aligned}
& \Gamma_{1} B_{r}\left(t_{i}\right) \subseteq\left\{T\left(t_{i}\right) u_{0}\right\}+\sum_{j=1}^{i} U\left(t_{i}-t_{j}, r\right) \quad \mathrm{e} \\
& \widetilde{\Gamma_{1} B_{r}}\left(t_{i}\right) \subseteq\left\{T\left(t_{i}\right) u_{0}\right\}+\sum_{j=i}^{i} U\left(t_{i}-t_{j}, r\right),
\end{aligned}
$$

o que implica que $\Gamma_{1} B\left(t_{i}\right)$ e $\overline{\Gamma_{1} B}\left(t_{i}\right)$ são relativamente compactos $\mathrm{cm} X$.

Pelo 'Icorema 3.4 conclúnos que $\Gamma_{1} B_{r}$ é relativamente compacto em $P C([0, T]: X)$. Portanto $\Gamma_{1}$ é um operador completamente contínuo.

Caso 2: $O$ conjunto $]_{2}\left(B_{r}\right)$ ć relativamente compacto em $P C([0, T]: X)$.

Como $\Gamma_{2}\left(B_{r}\right)$ é um conjunto de funções contínuas, podemos usar o clássico Teorema de Áscoli-Arzelá para mostrar a propriexdade. Estudanos primeiro a equicontinuidade de $\Gamma_{2}\left(B_{r}\right)$. Sejam $\epsilon>0$ e $t<t^{\prime}<T$. Por hipótese, sabcmos que existe um compacto $U(\delta, r)$ tal que $\left\{T(\delta) f(s, x, y): s \in[0, T], x, y \in B_{r}(0, X)\right\} \subset U(\delta, r)$. Usando a continuidade de $m(\cdot)$ e o Lema 2.17 , escolhemos $\delta>0$ tal que.

$$
\begin{aligned}
\int_{s}^{s^{\prime}} m(s) d s & <\frac{\epsilon}{6 M W(2 r)} ; \\
\|\left(T(s)-T\left(s^{\prime}\right)\right) x & <\frac{\epsilon}{3 M T}, \quad x \in U(\delta, r),
\end{aligned}
$$

se $s, s^{\prime} \in I$ e $\left|s-s^{\prime}\right| \leq \delta$. Nestas condiçōes, para $u \in B_{r}$ e $t \in I$ tal que $0<t^{\prime}-t \leq \delta$ temos que

$$
\begin{aligned}
\left\|\Gamma_{2} u(t)-\mathrm{I}_{2} u(t)\right\| \leq & \int_{0}^{t-\delta}\left\|\left(T(t-s-\delta)-T\left(t^{\prime}-s-\delta\right)\right) T(\delta) f(s, u(s), u(a(s)))\right\|^{i} d s \\
& +\int_{t-\delta}^{t}\left\|\left(T(t-s)-T\left(t^{\prime}-s\right)\right) f(s, u(s), u(a(s)))\right\| d s \\
& +\int_{t}^{t^{\prime}} \| T\left(t^{\prime}-s\right) f(s, u(s), u(a(s)) \| d s \\
\leq & \int_{0}^{t-\delta} \frac{\epsilon}{3 M T} d s+2 M \int_{t \delta}^{t} m(s) W(\|u(s)\|+\|u(a(s))\|) d s \\
& +M \int_{t}^{t^{\prime}} m(s) W(\|u(s)\|+\|u(a(s))\|) d s \\
< & \frac{c}{3 M T}(t-\delta)+2 M W(2 r) \int_{t \cdot \delta}^{t} m(s) d s+M W(2 r) \int_{t}^{t^{\prime}} m(s) d s \\
< & \epsilon,
\end{aligned}
$$


o que mostra que $\mathrm{I}_{2} B_{\mathrm{r}}$ é equicontínuo à esquerda $\mathrm{cm} t^{\prime} \in(0, T)$. A equicontinuidade à direita em $t^{\prime} \in(0, T)$ é provada usando argumentos auálogos. Isto completa a demonstração da equicontinuidade de $\Gamma_{2} B_{r}$.

Estudamos agora a compacidade do conjunto $\Gamma_{2} B_{r}(t)$. Como $\Gamma_{2} B_{r}(0)=\{0\}$, somente mostramos que $\Gamma_{2} B_{r}(t)$ é rclativamente compacto para todo $t \neq 0$. Sejam $0<\epsilon<t \leq T$ e $U(\epsilon, r) \subset X$ compacto tal que $\left\{T(\epsilon) f(s, x, y): s \in[0, T], x, y \in B_{r}(0, X)\right\} \subset U(\epsilon, r)$. Usando as consideraçǒes anteriores e o Teorema do Valor Médio para Integral de Bochner, veja Martin [4] pp. 25, temos que para $u \in B_{r}$

$$
\begin{aligned}
& \Gamma_{2} u(t)=\int_{0}^{t-\epsilon}\left(T(t-s-c) T(c) f(s, u(s), u(a(s))) d s+\int_{t-\epsilon}^{t} T(t-s) f(s, u(s), u(a(s))) d s\right. \\
& \in(l-\epsilon) \overline{\operatorname{com} v(\{T(s) x ; a \in U(\epsilon, r), s \in[0, T]\})}+C_{1},
\end{aligned}
$$

onde conv $(B)$ é a envolvente convexa de $B$ e $C_{1} \subset X$ é tal que diam $\left(C_{1}\right)<M \int_{t-\epsilon}^{b} m(s) d s$. Isto prova que $\Gamma_{2} B_{r}$ é um conjunto totalmente limitado em $X$ e portanto relativanente compacto em $X$, completando a prova de que $\Gamma_{2}$ é completamente contínuo.

É claro agora que $\Gamma$ verifica as condiçöes do 'Teorema 3.1. Portanto $\Gamma$ possui un ponto fixo em $\mathcal{P} C$ e conseqüentemente existe una solução fraca do problema impulsivo (3.1)-(3.3). Isto completa a prova.

Quando as funções $I_{i}$ são Lipschitz, o scguinte resultado é válido:

Teorema 3.6. Assuma que a condiçäo $\left(\mathbf{H}_{2}\right)$ do Teorema 3.5 se verifica. Suponha ainda que a seguinte propriedade é satisfeita:

$\left(\mathbf{H}_{3}\right)$ Existcm constantes positivas $L_{i}, i=1, \ldots, n$, tais que

$$
\left\|I_{i}(x)-I_{i}(y) \mid i \leq L_{i}\right\| x-y \|, \quad \forall(x, y) \in X^{2} .
$$

Se

$$
M \sum_{i=1}^{n} L_{i}+M \liminf _{r \rightarrow \infty} \frac{W(2 r)}{r} \int_{0}^{T} m(s) d s<1,
$$

então existe uma solução fraca do problema impulsivo (3.1)-(3.3).

Demonstração. Seja $\Gamma: \mathcal{P} C([0, T]: X) \rightarrow \mathcal{P} C([0, T]: X)$ a função definida em (3.4) e considere a decomposição $I^{\prime}=\Gamma_{1}+\Gamma_{2}$ introduzida em (3.6)-(3.7). Afirmamos que existe $r>0$ tal que $\Gamma\left(B_{r}(0, \mathcal{P} C)\right) \subset B_{r}(0, \mathcal{P} C)$ e $\Gamma_{i}\left(B_{r}(0, \mathcal{P} C)\right) \subset B_{r}(0, \mathcal{P} C)$, para $i=1,2$. De fato, se a afirmação é falsa, para cada $r>0$ existe $x_{r} \in B_{r}=B_{r}(0, \mathcal{P} C)$ tal que $\left\|\Gamma x_{r}\right\|_{T}>r$. Então

$$
r<\left\|\Gamma x_{r}\right\|_{T} \leq M\left\|u_{0}\right\|+M \sum_{i=1}^{n}\left(\left\|I_{i}\left(x_{r}\right)-I_{i}(0)\right\|+\left\|I_{i}(0)\right\|\right)+M W(2 r) \int_{0}^{T} m(s) d s
$$


de onde segue que

$$
1 \leq M \sum_{i=1}^{n} L_{i}+M \liminf _{r \rightarrow \infty} \frac{W(2 r)}{r} \int_{0}^{T} m(s) d s
$$

o que é absurdo. Isto prova que existe $r>0$ tal que $\Gamma\left(B_{r}\right) \subset B_{r}$. Mais ainda, é fácil obscrvar das estimativas que $r$ pode ser escolhido tal que $\Gamma_{i}\left(B_{r}\right) \subseteq B_{r}, i=1,2$.

Mostramos agora que $\Gamma=\Gamma_{1}+\Gamma_{2}: B_{r} \rightarrow B_{r}$ verifica as hipóteses do Teorema 3.2. Pela prova do Teorema 3.5 , o operador $\Gamma_{2}: B_{r} \rightarrow B_{r}$ é completamente contínuo. Usando $\left(\mathbf{H}_{3}\right)$ venuos que para. $x, y \in B_{r}$

$$
\begin{aligned}
\left\|\Gamma_{1} x(t)-\Gamma_{1} y(t)\right\| & \leq \sum_{i=1}^{n} M L_{i}\left\|x\left(t_{i}\right)-y\left(t_{i}\right)\right\| \\
& \leq\left(\sum_{i=1}^{n} M L_{i}\right)\|x-y\|_{T}
\end{aligned}
$$

e então

$$
\left\|\Gamma_{1} x-\Gamma_{1} y\right\|_{T} \leq\left(\sum_{i=1}^{n} M L_{i}\right)\|x-y\|_{T},
$$

o que mostra que $\Gamma_{1}: B_{r} \mapsto B_{r}$ é una contraçäo.

Portanto $\Gamma=\Gamma_{1}+\Gamma_{2}$ é um operador condensante em $B_{r}$ e, pelo Teorema $3.2, \Gamma$ possui um ponto fixo en $B_{r}$. Este ponto fixo é uma solução fraca do problema (3.1)-(3.3). Agora a prova do teorema está completa.

O próximo resultado é uma consequência direta do Teorema 3.6 e por isso onitimos a demonstração.

Corolário 3.7. Suponha que a condiçâo $\left(\mathbf{H}_{2}\right)$ do Teorema 3.5 se verifica e que cada fungão $I_{i}$ é uma transformação linear contínua em $X$.

Se

$$
M \sum_{i-1}^{n}\left\|I_{i}\right\|_{L(x)}+M \liminf _{r \rightarrow \infty} \frac{W(2 r)}{r} \int_{0}^{T} m(s) d s<1,
$$

entầo existe uma soluçäo fraca de (3.1)-(3.3).

\subsection{Existência de soluções globais}

Nesta parte do trabalho estudarnos a existéncia de soluçôes globais para o problema impulsivo

$$
\begin{aligned}
\dot{u}(t) & =A u(t)+f(t, u(t), u(a(t))), \quad t>0, \\
u(0) & =u_{0} \\
\Delta u\left(t_{i}\right) & =I_{u}\left(u\left(t_{i}\right)\right) \quad 0<t_{1}<t_{2}<\ldots<t_{n}<\ldots,
\end{aligned}
$$


onde: $A$ é o gerador de urn semigrupo for temente contínuo $(T(t))_{t \geq 0} \mathrm{em} X,\left(t_{i}\right)_{i \in \mathbb{N}}$ é uma seqüência crescente e não limitada de números reais positivos e $f:[0, \infty) \times X^{2} \rightarrow X$ é uma função contínua.

Para estudar a existência de solução para (3.10)-(3.12), definimos uma função $g:[0, \infty) \rightarrow \mathbb{E}$ positiva, contímua, näo decrescente, com $g(0)=1$ e tal que $g(t) \rightarrow \infty$ quando $t \rightarrow \infty$ e usamos as notaçoes $\mathcal{P} C([0, \infty): X), C_{g}^{0}(X)$ e $(\mathcal{P} C)_{g}^{0}(X)$ para os espaços:

$$
\begin{gathered}
\mathcal{P} C([0, \infty): X)=\left\{x:[0, \infty) \rightarrow X:\left.x\right|_{[0, T]} \in \mathcal{P} C([0, T]: X), \forall T, T \neq t_{i}\right\} \\
C_{g}^{0}(X)=\left\{x \in C([0, \infty): X): \lim _{t ; \infty} \frac{\|x(t)\|}{g(t)}=0\right\}, \\
(\mathcal{P} C)_{g}^{0}(X)=\left\{x \in \mathcal{P} C([0, \infty): X): \lim _{t \rightarrow \infty} \frac{\|x(t)\|}{g(t)}=0\right\},
\end{gathered}
$$

munidos das normas

$$
\|x\|_{x}=\sup _{t \geq 0}\|x(t)\|, \quad \forall x \|_{g}=\sup _{l \geq 0} \frac{\|x(t)\|}{g(t)} \quad \text { e } \quad\|x\| p_{y}=\sup _{l \geq 0} \frac{\| x(t)}{g(t)} \|_{i},
$$

respectivamente. É fácil mostrar que estes espaços sào espaços de Banach. Além disso,o seguinte resultado é válido:

Teorema 3.8. Um conjunto $B \subset C_{g}^{0}(X)$ é relativamente compacto se, e somente se:

(a) Bé equicontinno;

(b) $\frac{\|: x(t)\|}{g(l)} \rightarrow 0$ uniformemenle para $x \in B$ quando $t \rightarrow \infty$;

(c) $B(t)=\{x(t): x \in B\}$ é relativamente compacto em $X$ para todo $t \geq 0$.

Para $B \subset(P C)_{g}^{0}(X)$ e $T>0$ com $T \neq t_{i}$, usaremos a notação $B_{T}$ para o conjunto $B_{T}-$ $\left\{u_{T}=\left.u\right|_{[0, T]}: u \in B\right\}$. Observe que $B_{T^{\prime}} \subset \mathcal{P} C([0, T]: X)$.

Para mostrar a existência de soluçóes para o problema (3.10)-(3.12), usaremos o Teorema 3.1. Por esta razão consideramos o seguinte resultado:

Teorema 3.9. Um conjunlo $B C(P C)_{g}^{0}(X)$ é relativamente compacto se, e somente se:

(a) $B_{T}$ é relativamente compacto em $\mathcal{P} C([0, T]: X)$ para todo $T>0$;

(b) $\frac{\|x(t)\|}{g(t)} \rightarrow 0$ uniformemente para $x \in B$ : quando $t \rightarrow \infty$ 
Demonstração. Suponba que $B \subset(P C)_{g}^{0}(X)$ é relativanente compacto. A propriedade (a) é clara, pois $i_{T}:(\mathcal{P} C)_{g}^{0}(X) \rightarrow \mathcal{P} C([0, T]: X), u \mapsto u_{T}$, é contínua. Se a condição (b) é falsa, cntāo existe $\epsilon>0$ tal que para cada $n \in \mathbb{N}$ existem $x_{n} \in B$ e $s_{n} \geq n$ tais que

$$
\frac{\left\|x_{n}\left(s_{n}\right)\right\|}{g\left(s_{n}\right)} \geqslant c
$$

Usando a compacidade de $B$, podemos assumir: passando por subscqüencias se necessário, que $\left(x_{n}\right)_{\in \mathbb{N}}$ converge a uma função $x \in(P C)_{g}^{0}(X)$. Em particular, existe $N_{c} \in \mathbb{N}$ tal que.

$$
\| x_{n}-x i_{i p_{9}}<\frac{\epsilon}{2}
$$

quando $n \geq N_{\epsilon}$. Então para $n>N_{\epsilon}$ temos

$$
\frac{\left\|x\left(s_{n}\right)\right\|}{g\left(s_{n}\right)} \geq \frac{\left\|x_{n}\left(s_{n}\right)\right\|}{g\left(s_{n}\right)}-\frac{\left\|x\left(s_{n}\right)-x_{n}\left(s_{n}\right)\right\|}{g\left(s_{n}\right)} \geq \epsilon-\frac{\epsilon}{2}=\frac{\epsilon}{2}
$$

e portanto

$$
\liminf _{n \rightarrow \infty} \frac{\left|x\left(t_{n}\right)\right| \mid}{g\left(t_{n}\right)} \geq \stackrel{\epsilon}{2}
$$

o que é absurdo, pois $x \in\left(I^{P} C\right)_{g}^{0}(X)$. Isto prova que a condição (b) é satisfeita.

Suponha agora que as condiçoes (a), (b) são verificadas. Scja $\left(x_{n}\right)_{n \in \mathbb{N}}$ uma sequiência em $B$. Como para todo $T>0$ o conjunto $B_{T}$ ć relativamente compacto en $\mathcal{P} C([0, T]: X)$, podemos, usando um processo de diagonalização, achar uma subsecüiencia de $\left(x_{n}\right)_{n \in \mathbb{N}}$, que para evitar notações excessivas continuamos denotando do mesmo modo, e uma função $x \in \mathcal{P} C([0, \infty): X)$ tais que $\left(x_{n}\right)_{T} \rightarrow x_{T}$ em $\mathcal{P} C([0, T]: X)$ para todo $T>0$. Afirmamos que $x \in(\mathcal{P} C)_{g}^{0}(X) \mathrm{e}$ que $\left(x_{n}\right)_{\pi \in \mathbb{N}}$ converge a $x$ en $(\mathcal{P} C)_{g}^{0}(X)$. Mostramos primeiro que $x \in(\mathcal{P} C)_{g}^{0}(X)$. Scja $c>0$ e fixemos $T>0$ tal que $\frac{\|u(t)\|}{g(t)}<i, u \in B$, quando $t>T$. Como $x_{n} \rightarrow x$ uniformonente em compactos, para cada $t \geq 0$ existe $N_{l, r}$ tal que $\left\|x_{n}(t)-x(t)\right\|<\epsilon$ para todo $n \geq N_{t, c}$. Nestas condiçoes, para $t>T$

$$
\begin{aligned}
\frac{\|x(t)\|}{g(l)} & \leq \frac{\left\|x(t)-x_{N_{t, c}}(t)\right\|}{g(t)}+\frac{\left\|x_{N_{t, c}}(t)\right\|}{g(t)} \\
& \leq \| x(t)-x_{N_{t, c}}(t)+\frac{\left\|x_{N_{t, c}}(t)\right\|}{g(t)} \\
& <\epsilon .
\end{aligned}
$$

Assim

$$
\frac{\|x(t)\|}{g(t)}<\epsilon, \text { para } t>T
$$

e portanto $x \in(\mathcal{P C})_{g}^{0}(X)$. Mostramos agora a convergencia. Sejann $\epsilon>0$ e $T_{1}>0$ tais que

$$
\sup \left\{\frac{\| x(t)}{g(t)} \| \frac{\left\|x_{n}(t)\right\|}{g(t)}: n \in \mathbb{N}, t \geq T_{1}\right\} \leq \frac{\epsilon}{2}
$$


Usando que $\left(x_{n}\right)_{T_{1}} \rightarrow x_{T_{1}}$ em $\mathcal{P C}\left(\left[0, T_{1}\right]: X\right)$, escolhemos $N_{\mathrm{t}} \in \mathbb{N}$ tal que

$$
\sup \left\{\left\|x(t)-x_{n}(t)\right\|: t \in[0, T], n \geq N_{\epsilon}\right\}<\frac{\epsilon}{2}
$$

Segue de (3.13) e (3.14) que para $u>N_{t}$

$$
\begin{aligned}
\left\|x_{n}-x\right\|_{1} & \leq \sup \left\{\frac{\left\|x_{n}(t)-x(t)\right\|}{g(t)}: t \in\left[0, T_{1}\right]\right\}+\sup \left\{\frac{\left\|x_{n}(t)-x(t)\right\|}{g(t)}: t \geq T_{1}\right\} \\
& \leq \sup \left\{\left\|x_{n}(t)-x(t)\right\|: t \in\left[0, T_{1}\right]\right\}+\frac{\epsilon}{2} \\
& <\epsilon,
\end{aligned}
$$

o que mostra que $x_{n} \rightarrow x$ em $(\mathcal{P} C)_{g}^{0}(X)$. Como $\left(x_{n}\right)_{n \in \mathbb{N}}$ é uma seqüência arbitrária em $B$, concluímos que $B$ é relativamente compacto em $(\mathcal{P C})_{g}^{1 !}(X)$. $\Lambda$ prova do teorema está completa.

Apresentamos agora um primciro resultado de existência de soluções globais.

Teorema 3.10. Suponha que as seguintes propriedades säo verificadas:

$\left(\tilde{\mathbf{H}}_{1}\right)$ As funçöes $I_{i}, i \in \mathbb{N}$, säo completamente contínuas, limitadas $e \sum_{i=1}^{\infty} N_{i}<\infty$, onde $N_{i}=$ $\sup \left\{\left\|I_{i}(x)\right\|: x \in X\right\}$;

$\left(\tilde{\mathbf{H}}_{2}\right)$ Para todo $t>0$ e $r>0$ o conjunto $\left\{T(t) f(s, x, y): s \in[0, t], x, y \in B_{r}(0, X)\right\}$ é relativamente compacto em $X$;

$\left(\mathbf{H}_{4}\right)$ Para todo $K>0, \frac{1}{g(t)} \int_{0}^{t} m(s) W(K g(s)) d s \rightarrow 0$ quando $t \rightarrow \infty$.

Sejac $-M\left\|x_{0}\right\|+M \sum_{i=1}^{\infty} N_{i} . \quad$ Se $2 M \int_{0}^{\infty} m(s) d s<\int_{c}^{\infty} \frac{1}{2 W(s)} d s$, então cxiste uma soluçäo fraca do problema (3.10)-(3.1\%).

Demonstraçāo. Para uma função $u \in(\mathcal{P} C)_{g}^{0}(X)$ definimos $\Gamma u \in(\mathcal{P} C)([0, \infty): X)$ por

$$
\Gamma u(t)=T(t) u_{0}+\int_{0}^{t} T(t-s) f(s, u(s), u(a(s))) d s+\sum_{t_{i}<t} T\left(t-t_{i}\right) I_{i}\left(u\left(t_{i}\right)\right) .
$$

Usando que $\|u(t)\| \leq i \mid u \|_{g} g(t)$, da definição de I'u vemos que

$$
\begin{aligned}
\frac{\|\Gamma u(t)\| \leq}{g(t)} \leq & \frac{\left\|T(t) u_{0}\right\|}{g(t)}+\frac{M}{g(t)} \int_{0}^{t} m(s) W(\|u(s)\|+\|u(a(s))\|) d s \\
& +\frac{1}{g(t)} \sum_{t_{i}<t}\left\|T\left(t-t_{t}\right)\right\| N_{i} \\
\leq & \frac{M\left\|u_{0}\right\|}{g(t)}+\frac{M}{g(t)} \int_{0}^{t} m(s) W\left(\|u\|_{g} g(s)+\|u\|_{g} g(a(s))\right) d s+\frac{M}{g(t)} \sum_{t_{i}<t} N_{i} \\
\leq & \frac{M\left\|u_{0}\right\|}{g(l)}+\frac{M}{g(t)} \int_{0}^{t} m(s) W\left(2\|u\|_{g} g(s)\right) d s+\frac{M}{g(t)} \sum_{i=1}^{\infty} N_{i},
\end{aligned}
$$




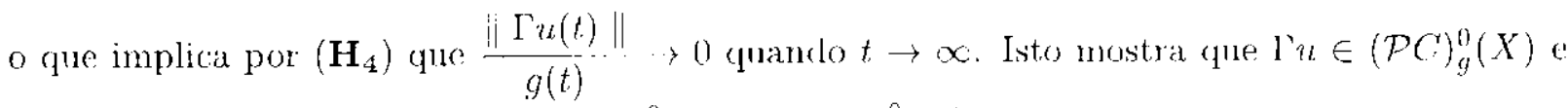
portanto $\Gamma$ define uma função de $(P C)_{g}^{0}(X)$ erm $(\mathcal{P C})_{g}^{0}(X)$.

Para provar que I possui un ponto fixo, mostramos que $\Gamma$ vorifica as hipóteses do Teorema 3.1. Vejamos inicialmente a continuidade. Sejam $\left(x_{n}\right)_{n \in \mathbb{N}}$ una seqüencia em $(P C)_{g}^{0}(X)$ e $x \subset$ $(P C)_{g}^{0}(X)$ tais que $x_{n} \rightarrow x$ em $(P C)_{g}^{0}(X)$. Para $\epsilon>0$, fixemos $L>0$ tal que

$$
\frac{M}{g(t)} \int_{0}^{t} m(s) W(2 K g(s)) d s+\frac{2 M}{g(t)} \sum_{i=1}^{\infty} N_{i}<\frac{c}{3}, \quad t \geq L_{,}
$$

onde $K=\sup \left\{\left\|x_{n} \quad\right\|_{g},: x \|_{g}: n \in \mathbb{N}\right\}$. Cono $f$ é contímia, do Teorema da convergêneia dominada de Lebesgue e de $\left(\mathbf{A}_{2}\right)$, temos que existe $N_{c}^{1} \in \mathbb{N}$ tal que para todo $n \geq N_{\epsilon}^{1}$

$$
\int_{0}^{l}\left\|f\left(s, x_{n}(s), x_{n}(a(s))\right) d s-\int_{0}^{l} f(s, x(s), x(a(s)))\right\| d s<\frac{\epsilon}{3 M} .
$$

Usando agora a continuidade das funçocs $I_{i}$ e o fato de que $x_{n} \rightarrow x$ uniformemente em compactos de $\left[0, \infty^{+}\right)$, escolhemos $N_{\epsilon}^{2}$ tal que para todo $n>N_{\varepsilon}^{2}$

$$
\sum_{t_{i} \leq L}\left\|I_{i}\left(x_{n}\left(t_{i}\right)\right)-I_{i}\left(x\left(t_{i}\right)\right)\right\|<\frac{\epsilon}{3 M}
$$

Usando (3.17) e (3.18) scgue que para $n \geq N_{\epsilon}^{3}=\max \left\{N_{\epsilon}^{1}, N_{\epsilon}^{2}\right\}$ e $t \in[0, L]$,

$$
\begin{aligned}
\frac{\left\|\Gamma x_{n}(t)-\Gamma x(t)\right\|}{g(t)} \leq & \left.\frac{M}{g(t)} \int_{0}^{t} \|\right)\left(f\left(s, x_{n}(s), x_{n}(a(s))\right)-f(t, x(s), x(a(s)))\right) \| d s \\
& +\frac{1}{g(t)} \sum_{t_{i} \leq L} M\left\|I_{i}\left(x_{n}\left(t_{i}\right)\right)-J_{i}\left(x\left(t_{i}\right)\right)\right\| \\
< & \epsilon
\end{aligned}
$$

e portanto

$$
\sup \left\{\frac{\| x_{n}(t)-\Gamma x(t) ! \mid}{g(\ell)}: t \in[0, L]: n \geq N_{\epsilon}^{3}\right\} \leq \iota
$$

Por outro lado, de (3.16), (3.17) e (3.18) temos que para $t \geq L$ e $n \geq N_{\epsilon}^{3}$

$$
\begin{aligned}
\frac{\left\|\Gamma x_{n}(t)-\Gamma x(t)\right\| \leq \leq}{g(t)} \| & \frac{1}{g(t)} \int_{0}^{L}\left\|T(t-s)\left(f\left(s, x_{n}(s), x_{n}(a(s))\right)-f(s, x(s), x(a(s)))\right)\right\| d s \\
& +\frac{1}{g(t)} \int_{L}^{t}\left\|T(t-s)\left(f\left(s, x_{n}(s), x_{n}(a(s))\right)-f(s, x(s), x(a(s)))\right)\right\| d s \\
& +\frac{M}{g(t)} \sum_{t_{i} \leq L}\left\|I_{i}\left(x_{n}\left(t_{i}\right)\right)-I_{i}\left(x\left(t_{i}\right)\right)\right\|+\frac{1}{g(t)} \sum_{t_{i} \geq L} 2 M N_{i} \\
\leq & \frac{\epsilon}{3}+\frac{M}{g(t)} \int_{L}^{t} m(s) W(2 K g(s)) d s+\frac{1}{g(t)} \sum_{t_{i} \geq L} 2 M N_{i}+\frac{\epsilon}{3} \\
< & \epsilon
\end{aligned}
$$


e assim

$$
\sup \left\{\frac{\left\|x_{n}(t)-\Gamma x(t)\right\|}{g(t)}: t \geq L, n \geq N_{t}^{3}\right\} \leq \epsilon .
$$

Segue agora de (3.19) e (3.20) que

$$
\left\|\Gamma x_{n}-\Gamma x\right\|_{9} \leq \epsilon
$$

quando $n \geq N_{t}^{3}$. Portanto $\Gamma$ é contínua.

Mostramos agora que $\Gamma$ é completamente contínua usando o Teorema 3.9. Sejam $r>0$ o $B_{r}=B_{r}\left(0,(\mathcal{P C})_{g}^{0}(X)\right)$. Usando as mesmas idéias e estimativas da demonstração do Teorema 3.5 vemos que $\Gamma\left(B_{r}\right)_{1}$ é relativamente compacto em $\mathcal{P} C([0, T]: X)$ para todo $T>0, T \neq t_{i}$. Mais ainda, como para $x \in B_{r}$

$$
\frac{\|\Gamma(t)\|}{g(t)} \leq \frac{M\left\|u_{0}\right\|}{g(t)}+\frac{M}{g(t)} \int_{0}^{t} m(s) W\left(2\|x\|_{g} g(s)\right) d s+\frac{M}{g(t)} \sum_{i \cdots 1}^{\infty} N_{i},
$$

segue de $\left(\mathbf{H}_{\mathbf{4}}\right)$ que

$$
\frac{\|\Gamma x(t)\|}{g(t)} \rightarrow 0
$$

uniformemente para $x \in B_{r}$ quando $t \rightarrow \infty$. Assim $\Gamma\left(B_{r}\right)$ verilica as hipóteses do T'orema $3.9 \mathrm{e}$ portanto é relativamente compacto em $(P C)_{g}^{0}(X)$. Isto prova que $\Gamma$ é completamente contínua, pois $r$ é arbitrário.

Mostramos agora que o conjunto $U=\left\{x_{\lambda} \in(P C)_{g}^{0}(X): \lambda \Gamma x_{\lambda}=x_{\lambda}, \lambda \in(0,1)\right\}$ é limitado $\mathrm{em}(P C)_{g}^{0}(X)$. Se $x_{\lambda} \in U$, para $t \geq 0$ temos

$$
\begin{aligned}
\left\|x_{\lambda}(t)\right\| \leq & \left\|T(t) u_{0}\right\|+\int_{0}^{t} m(s) W\left(\left\|x_{\lambda}(s)\right\|+\left\|x_{\lambda}(a(s))\right\|\right) d s+\sum_{i=1}^{\infty} M N_{i} \\
\leq & M\left\|x_{0}\right\|+M \int_{0}^{t} m(s) W\left(\left\|x_{\lambda}(s)\right\|+\left\|x_{\lambda}(a(s))\right\| d s\right. \\
& +M \sum_{i=1}^{\infty} N_{i} .
\end{aligned}
$$

Denotando por $u_{\lambda}(l)$ o lado direito da última desigualdade obtemos

$$
\dot{u}_{\lambda}(t) \leq M m(t) W\left(2 u_{\lambda}(t)\right)
$$

e portanto

$$
\int_{2 u_{\lambda}(0)-c}^{2 u_{\lambda}(t)} \frac{1}{W(s)} d s \leq 2 M \int_{0}^{t} m(s) d s<\int_{c}^{\infty} \frac{1}{W(s)} d s .
$$

Como $x_{\lambda}$ e $\lambda$ säo arbitrários, é conseqüencia de (3.21) que o conjunto $\left\{u_{\lambda} ; \lambda \in(0,1)\right\}$ é linitado en $C([0, \infty): X)$, o que implica que $U$ é limitado cm $(P C)_{g}^{0}(X)$. 
Isso mostra que $\Gamma$ verifica as hipóteses do Teorema 3.9 e prova que existe una soluçáo fraca do problema (3.10)-(3.12). A prova está completa.

Teorema 3.11. Suponha que as condiçoes $\left(\tilde{\mathbf{H}}_{2}\right) \in\left(\mathbf{H}_{4}\right)$ säo verificadas. Supontha ainda que $\left(\tilde{\mathbf{H}}_{3}\right)$ Para cada $i \in \mathbb{N}$ existe $L_{i}>0$ tal que

$$
\left\|I_{i}(x)-I_{i}(y)\right\| \leq L_{i} \vdots x-y !: \quad \forall(x, y) \in X^{2}
$$

Se

$$
M \sum_{i=1}^{\infty} L_{i}+\limsup _{\mid(t, r) \rightarrow \infty} M \int_{0}^{t} m(s) \frac{W(2 r g(s))}{r g(s)} d s<1
$$

então existe uma solução fraca do problema (3.10)-(3.12).

Demonstração. Para $u \in(\mathcal{P} C)_{g}^{0}(X)$ definimos I" $:(\mathcal{P} C)_{g}^{0}(X) \rightarrow(\mathcal{P C})([0, \infty): X)$, onde

$$
\Gamma u(t)=T(t) u_{0}+\int_{0}^{t} T(t-s) f(s, u(s), u(a(s))) d s+\sum_{t_{2}<t} T\left(t-t_{i}\right) I_{i}\left(u\left(t_{i}\right)\right) .
$$

Da prova do Teorena 3.10 sabcmos que $\Gamma u \in(\mathcal{P} C)_{g}^{0}(X)$ e que $\Gamma:(\mathcal{P} C)_{g}^{0}(X) \rightarrow(\mathcal{P} C)_{g}^{0}(X)$ é contínua. Considere a decomposição $\mathrm{I}^{\prime}=\mathrm{l}_{1}{ }_{1}+\Gamma_{2}$, onde

$$
\Gamma_{1} u(t)-T(t) u_{0}+\sum_{t_{i}<t} T\left(t-t_{i}\right) I_{i}\left(u\left(t_{i}\right)\right)
$$

Seja $B_{r}=B_{r}\left(0,(\mathcal{P} C)_{g}^{0}(X)\right)$. Afirmamos que existe $r>0$ tal que I' $B_{r} \subset B_{r}$ e $\Gamma_{i} B_{r} \subset B_{r}, i=1,2$. De fato, se a nossa afirmação é falsa então para cadá $r>0$ existem $x_{r} \in B_{r}$ e $t^{r}>0$ tais que $r<\left\|\Gamma x_{r}\left(t^{r}\right)\right\|$. Logo

$$
\begin{aligned}
r<\frac{\left\|\Gamma x_{r}\left(t^{r}\right)\right\| \leq}{g\left(t^{r}\right)} \leq & \frac{M\left\|u_{0}\right\|}{g\left(t^{r}\right)}+\frac{M}{g\left(t^{r}\right)} \int_{0}^{t^{r}} m(s) W\left(\left\|x_{r}(s)\right\|+\left\|x_{r}(a(s))\right\|\right) d s \\
& +\frac{M}{g\left(t^{r}\right)} \sum_{t_{i}<t^{r}}\left(I_{i}\left\|x_{r}\left(t_{i}\right)\right\|+\left\|I_{i}(0)\right\|\right) \\
\leq & \frac{M\left\|u_{0}\right\|^{\prime}}{g\left(t^{r}\right)}+\frac{M}{g\left(t^{r}\right)} \int_{0}^{t^{r}} m(s) W(2 r g(s)) d s+\frac{M r g\left(t_{i}\right)}{g\left(t^{r}\right)} \sum_{t_{i}<t^{r}} L_{i} \\
& +\frac{M}{g\left(t^{r}\right)} \sum_{t_{i}<t^{r}}\left\|I_{i}(0)\right\| .
\end{aligned}
$$

Dependendo de o conjunto $U=\left\{t^{r}: r>0\right\}$ ser limitado ou não, da desigualdade anterior obtemos

$$
\left\{\begin{array}{l}
1 \leq M \sum_{i=1}^{n} L_{i}, \text { se } U \text { é limitado } \\
1 \leq M \sum_{i=1}^{\infty} L_{i}+\lim \sup _{(t, r) \mid \rightarrow \infty} M \int_{0}^{t} m(s) \frac{W(2 r g(s))}{r g(s)} d s, \text { se } U \text { não é limitado, }
\end{array}\right.
$$


o que é absurdo. Isto prova que existe $r_{0}$ tal que $\Gamma B_{r_{0}} \subset B_{r_{0}}$. Mais ainda, a mesma estimativa mostra que é possível assumir que $\Gamma_{2} B_{r_{0}} \subset B_{r_{0}}, i=1,2$.

Usando a prova do Teorema 3.10 , vemos que $\mathrm{I}_{2}$ é completamente contínua em $B_{r_{0}}$. Além disso, $\Gamma_{1}$ é uma contraçăo em $B_{r_{0}}$. De fato, se $x, y \in B_{r}$ temos

$$
\begin{aligned}
\frac{\left\|\Gamma_{1} x(t)-\Gamma_{1} y(t)\right\|}{g(t)} & \leq \frac{M}{g(t)} \sum_{t_{2}<t}\left\|L_{i}\right\| x\left(t_{i}\right)-y\left(t_{i}\right) \| \\
& \leq \frac{M}{g(t)} \sum_{t_{i}<t} g\left(t_{i}\right)\left\|L_{i}\right\|: x-y \|_{g}
\end{aligned}
$$

e então

$$
\left\|\Gamma_{1} x-\Gamma_{1} y\right\|_{g} \leq M \sum_{i-1}^{\infty}\left\|L_{i}\right\|\|x-y\|_{g} .
$$

Segue agora de (3.22) e (3.23) que $\Gamma_{1}$ é una contração em $B_{r_{0}}$.

Das consideraçöes anteriores, deduzimos que $\Gamma$ é uma função condensante cm $B_{r_{0}}$. A existência de uma soluçâo fraca do problema (3.10)-(3.12) é agora conseqụ̈̂ncia do Teorema 3.2. A prova está completa.

Teorema 3.12. Suponha que as condiçoes $\left(\overline{\mathbf{H}}_{2}\right)$ e $\left(\mathbf{H}_{4}\right)$ säo verificadas. Assuma também que: $\left(\tilde{\mathbf{H}}_{1}^{\prime}\right)$ Cada funçäo $I_{i}$ é um operador linear contínuo;

$\left(\mathbf{H}_{5}\right)$ Para cada $s \geq 0, f(s, \cdot)=L(s, \cdot)+h(s)$. onde $L(s, \cdot)$ é um operador linear limitado e $h(\cdot)$ é uma funçû́o contínua tal que $\frac{1}{g(t)} \int_{0}^{t} h(s) d s \rightarrow 0$ quando $t \rightarrow \infty$.

Se $M \sum_{i=1}^{\infty} L_{i}+M \sup _{t \geq 0} \frac{1}{g(t)} \int_{0}^{t}\|L(s)\| g(s) d s<1$, entäo existe uma única solução fraca do problema (3.10)-(3.12).

Demonstração. Seja $\Gamma:(\mathcal{P C})_{g}^{0}(X) \rightarrow(\mathcal{P C})([0, \infty): X)$ a função definida na demonstraçăo do Tcorcma 3.10, veja (3.15). Sabemos da prova do Teorema 3.10 que l'é uma função bem definida, contínua e com valores em $(\mathcal{P} C)_{g}^{0}(X)$. Mostramos agora que $\Gamma$ é uma contração erı $(\mathcal{P} C)_{g}^{0}(X)$. Fixemos $x, y \in(\mathcal{P} C)_{g}^{0}(X)$. Se $t \geq 0$ temos

$$
\begin{aligned}
\frac{\|\Gamma x(t)-\Gamma y(t)\|}{g(t)} \| & \leq \frac{M}{g(t)} \sum_{t_{i}<t}\left\|I_{\imath}\right\|\left\|x\left(t_{i}\right)-y\left(t_{i}\right)\right\|+\frac{M}{g(t)} \int_{0}^{t}\|L(s)\| x(s)-y(s) \| d s \\
& \leq \frac{M g\left(t_{i}\right)}{g(t)} \sum_{t_{i}<t}\left\|I_{i}\right\|\|x-y\|_{g}+\|x-y\|_{g} \frac{M}{g(t)} \int_{0}^{t}\|L(s)\| g(s) d s \\
& \leq\left(M \sum_{t_{i}<t}\left\|I_{i}\right\|+M \int_{0}^{t}\|L(s)\| d s\right)\|x-y\|_{g}
\end{aligned}
$$


c portanto

$$
\|\Gamma x-\Gamma y\|_{g} \leq\left(M \sum_{i=1}^{\infty}\left\|I_{i}\right\|+\frac{M}{g(t)} \int_{0}^{t}\|L(s)\| g(s) d s\right)\|x-y\|_{g}
$$

o que implica que $\Gamma$ é uma contração.

Pelo teorema de ponto tixo para contraçoes, I possui uru único ponto fixo $x \subset(\mathcal{P C})_{g}^{0}(X)$. Este ponto fixo é a única solução fraca do problema (3.10)-(3.12). A prova está completa.

O resultado seguinte é uma conseqüência óbvia do teorema anterior.

Corolário 3.13. Assuma que se verificam as condições $\tilde{\mathbf{H}}_{\mathbf{1}}^{\prime}, \tilde{\mathbf{H}}_{\mathbf{2}}, \mathbf{H}_{\mathbf{4}}, \mathbf{H}_{\mathbf{5}}$. Se

$$
M \sum_{i=1}^{\infty} L_{i}+M \int_{0}^{\infty} \|\left. L(s)\right|_{i} d s<1
$$

então existe uma única soluçäo fraca do problema (3.10)-(3.12).

\subsection{Um exemplo: A equação do calor com impulsos}

Para finalizar este trabalho, consideramos brevenente um problema que estamos chamando de equação do calor com impulsos. Scjam $X=L^{2}([0, \pi])$, munido da norma usual que denotaremos $\|\left.\cdot\right|_{2}$, e $A$ o operator $A x=x^{\prime \prime}$ com domínio

$$
D(A):=\left\{f \in L^{2}([0, \pi]): f^{\prime \prime} \in L^{2}([0, \pi]), f(0)=f(\pi)=0\right\} .
$$

Nestas condiçōes, $A$ ć o gerador de um $C_{0}$-semigrupo $(T(t))_{t \geq 0} \mathrm{em} X$. Mais ainda, $A$ tem espectro discreto, os autovalores são $-n^{2}, n \in \mathbb{N}$, com os autovetores correspondentes $z_{n}(\xi):=$ $(2 / \pi)^{1 / 2} \operatorname{sen}(n \xi)$ e as seguintes propriedades são válidas:

(a) $\left\{z_{n}: n \in \mathbb{N}\right\}$ é uma base ortonormal de $X$;

(b) Se $f \in D(A)$ então $A(f)=-\sum_{\pi_{i=1}}^{\infty} n^{2}\left\langle f \cdot z_{n}\right\rangle z_{n}$;

(c) Para cada $f \in X, T(t) f=\sum_{n-1}^{\infty} e^{n^{2} t}\left(f, z_{n}\right) z_{n}$. Mais ainda, segue das expressóes anteriores que $\|T(t)\| \leq e^{-t} \leq 1$ para todo $t \geq 0$.

Consideremos agora o problema impulsivo

$$
\begin{aligned}
\frac{\partial}{\partial t} u(\xi, t) & =\frac{\partial^{2}}{\partial \xi^{2}} u(\xi, t)+F(\xi, t, w(\xi, t), w(\xi, a(t))), \\
w(0, t) & =w(\pi, t)=0, \quad t \in I, \\
w\left(\xi, t_{i}\right) & =P_{i}\left(u\left(\cdot, t_{i}\right)\right)(\xi), \quad \xi \in[0, \pi],
\end{aligned}
$$

onde $0<t_{1}<t_{2}<\ldots<t_{n}<\pi$ e 
(1) A função $F:\left[0, \pi j \times I \times \mathbb{R}^{2} \rightarrow \mathbb{R}\right.$ verifica as seguintes propriedades:

(a) $F(\xi, l, \cdot): \mathbb{R}^{2} \rightarrow \mathbb{R}$ é contínua q.t.p. em $(\xi, l) \in[0, \pi] \times I$.

(b) Para todo $w_{1}, w_{2} \subset \mathbb{R}$, a função $F\left(\cdot, w_{1}, w_{2}\right):[0, \pi] \times I \rightarrow \mathbb{R}$ é integrável.

(c) Existe uma função integrável e positiva $\eta(\cdot)$, definida sobre $[0, \pi] \times \mathbb{R}$ tal que

$$
\left|F\left(\xi, t, w_{1}, w_{2}\right)\right| \leq \eta(\xi, t)\left(\left|w_{1}\right|+\left|w_{2}\right|\right)
$$

para todo $w_{1}, w_{2} \in \mathbb{R}$.

(2) As funçôes $P_{i}: X \rightarrow X$ são completamente continuas. Assumimos ainda que para cada $r>0$ existem constantes $\alpha_{i, r}>0$ tais que $\left\|P_{i}(x)\right\|_{2} \leq \alpha_{i, r}$. Exemplos de operadores com estas propriedades podem ser encontrados em Pazy [1].

Seja $f:[0, T] \times X^{2} \rightarrow X$ o operador definido por $f(t, x, y)(\xi)=F(\xi, t, x(\xi), y(\xi))$. Nestas condiçoes, para $x, y \subset X,\|x\|_{2},\|y\|_{2} \leq r$ tomus

$$
\begin{aligned}
\|f(t, x, y)\|_{2} & \leq\left(\int_{0}^{\pi}\left|F^{\prime}(\xi, t, x(\xi), y(\xi))\right|^{2} d \xi\right)^{\frac{1}{2}} \\
& \leq\left(\int_{0}^{\pi} \eta^{2}(\xi, t)(|x(\xi)|+|y(\xi)|)^{2} d \xi\right)^{\frac{1}{2}} \\
& \leq \eta(\cdot, t)_{0 ; \pi}\left(\|x\|_{2}+\|y\|_{2}\right) .
\end{aligned}
$$

onde $\eta(\cdot, s)_{0, \pi}=\sup _{\theta \in[0, \pi]} \eta(\theta, s)$.

Teorema 3.14. Suponha que as condições anteriores säo satisfeitas. Se

$$
2 \int_{0}^{T} \eta(\cdot, s)_{0, \pi} d s+\liminf _{r \rightarrow x:} \sum_{i=1}^{n} \frac{1}{r} \alpha_{i, r}<1,
$$

onde $\eta(\cdot, s)_{0, \pi}-\sup _{\theta \in[0, \pi]} \eta(\theta, s)$, cntäo existe uma soluçáa fraca do problema impulsivo (3.24)(3.26).

Demonstração. Seja $\Gamma: \mathcal{P} C \rightarrow \mathcal{P C}$ o operador definido em (3.4). Afirmamos que existe $r>0$ tal que $\Gamma\left(B_{r}\right) \subset B_{r}$, onde $B_{r}=B_{r}(0, \mathcal{P} C)$. Se a propriedade á falsa, para cada $r>0$ podernos escolher $x_{r} \in B_{r}$ tal que $r<\left\|\Gamma x_{r}\right\|$. Assim

$$
r<_{\|} \mid \Gamma x_{r}\|\leq\| x_{0} \|_{i}+2 r \int_{0}^{t} \eta(\cdot s)_{0, \pi} d s+\sum_{i=1}^{n} \alpha_{i, r}
$$

e portanto

$$
1 \leq 2 \int_{0}^{T} \eta(\cdot, s)_{0, \pi} d s+\liminf _{r \rightarrow \infty} \sum_{i=1}^{n} \frac{1}{r} \alpha_{i, r},
$$


o clue ć absurdo.

As mesmas idéias da demonstração do Teorema 3.5 mostram que I'é completamente contínuo em $B_{r}$. Agora a existencia de solnçóes para (3.24)-(3.26) ć conseqüencia do Teorema de ponto fixo de Schauder, veja [4]: pp 126. A prova está completa. 


\section{Referências Bibliográficas}

[1] Goldstein, Jerome A.. Semigroups of linear operators and applications. Oxford Mathematical Monographs. The Clarendon Press, Oxford University Press, New York, 1985.

[2] Granas A., M. Frigon. $\Lambda$ Topological methods in differential equations and inclusions. NATO Advanced Science Institutes Series C: Mathematical and Physical Sciences, 472. Khwer Academic: Publishers Group, Dordrecht, 1995.

[3] Liu, James H.. Nonlinear impulsive evolution equations. Dynam. Contin. Discrete Impuls. Systems 6 (1999), no. 1, 77-85.

[4] Martin, Robert II.. Jr. Nonlinear opcrators and differential equations in Banach spaces. Pure and Applied Mathematics. Wiley-Interseience [John Wiley \& Sons], New YorkLondon-Sydney, 1976.

[5] Pazy, $\Lambda$.. Semigroups of linear operators and applications to partial differential equations. Applied Mathematical Sciences, 44. Springer-Verlag, New York-Berlin, 1983.

[6] Rogovchenko, Yuri V.. Impulsive evolution systems: main results and now trends. Dynam. Contin. I)iscrete Impuls. Systems 3 (1997), no. 1, $57-88$.

[7] B. N. Saclovskii. On a fixed point. principle. Funct. Anal. Appl. 1 (1967) 74-76.

[8] Yosida, Kôsaku. Functional analysis. Dic Grundlehren der Mathematischen Wisscnschalten, Band 123 Acadenic Press, Inc., New York; Springer-Verlag, Berlin 1965. 